\title{
The Prediction of Broadband Shock-Associated Noise Including Propagation Effects
}

\author{
Steven A. E. Miller* \\ The National Aeronautics and Space Administration \\ Philip J. Morris ${ }^{\dagger}$ \\ The Pennsylvania State University
}

\begin{abstract}
An acoustic analogy is developed based on the Euler equations for broadband shockassociated noise (BBSAN) that directly incorporates the vector Green's function of the linearized Euler equations and a steady Reynolds-Averaged Navier-Stokes solution (SRANS) as the mean flow. The vector Green's function allows the BBSAN propagation through the jet shear layer to be determined. The large-scale coherent turbulence is modeled by two-point second order velocity cross-correlations. Turbulent length and time scales are related to the turbulent kinetic energy and dissipation. An adjoint vector Green's function solver is implemented to determine the vector Green's function based on a locally parallel mean flow at streamwise locations of the SRANS solution. However, the developed acoustic analogy could easily be based on any adjoint vector Green's function solver, such as one that makes no assumptions about the mean flow. The newly developed acoustic analogy can be simplified to one that uses the Green's function associated with the Helmholtz equation, which is consistent with the formulation of Morris and Miller (AIAAJ 2010). A large number of predictions are generated using three different nozzles over a wide range of fully expanded Mach numbers and jet stagnation temperatures. These predictions are compared with experimental data from multiple jet noise labs. In addition, two models for the so-called 'fine-scale' mixing noise are included in the comparisons. Improved BBSAN predictions are obtained relative to other models that do not include the propagation effects, especially in the upstream direction of the jet.
\end{abstract}

\section{Nomenclature}

$a_{m} \quad$ Factor to match inner- and outer-solution magnitudes

$a_{n m} \quad$ Amplitudes of components of second-order cross-correlations

$c \quad$ Speed of sound

$D \quad$ Nozzle exit diameter

$f_{i}^{v} \quad$ Unsteady force per unit volume associated with velocity fluctuations

$f_{i}^{a} \quad$ Unsteady force per unit volume associated with speed of sound fluctuations

$g$ Green's function of Lilley's equation

$H_{i}^{(j)} \quad$ Hankel functions of kind $j$ and order $i$

$J_{i}$

Bessel function of the first kind and order $i$

Turbulent kinetic energy

Spatial wavenumber vector component in the $i$ direction

Integral length scale in the streamwise direction

Integral length scale in the cross-stream direction

*Research Aerospace Engineer, Aeroacoustics Branch, NASA Langley Research Center, 2 N. Dryden St. MS 461, Hampton, VA, 23681, USA, AIAA Member, s.miller@nasa.gov

${ }^{\dagger}$ Boeing / A. D. Welliver Professor of Aerospace Engineering, The Pennsylvania State University, Aerospace Engineering, 229 Hammond Building, University Park, PA 16802, USA, AIAA Fellow, pjm@psu.edu 


\begin{tabular}{|c|c|}
\hline$M$ & Mach number \\
\hline$M_{d}$ & Design Mach number \\
\hline$M_{j}$ & Fully expanded Mach number \\
\hline$m$ & Azimuthal mode number \\
\hline$N P R$ & Nozzle pressure ratio \\
\hline$p$ & Pressure \\
\hline$p_{s}$ & Shock pressure perturbation \\
\hline$\tilde{p}_{s}$ & Fourier transform of the shock pressure perturbation in the streamwise direction \\
\hline$R$ & Distance from source point to observer \\
\hline$R_{o}$ & Radial location between the inner and outer solutions of the Green's function solver \\
\hline$R_{n m}$ & Two-point cross-correlation of the turbulent velocity fluctuations \\
\hline$R_{n m}^{v}$ & Two-point cross-correlation of $f_{n}^{v}$ \\
\hline$r$ & Radial direction \\
\hline$S$ & Spectral density \\
\hline$S_{n m}$ & Wavenumber frequency spectrum of the turbulent velocity correlation \\
\hline$T T R$ & Total temperature ratio \\
\hline$t$ & Time \\
\hline$u$ & Streamwise velocity \\
\hline$v$ & Radial velocity \\
\hline$v_{g i}^{n}$ & Component of the vector Green's function of the linearized Euler equations \\
\hline$x$ & Streamwise direction \\
\hline$\underline{x}=\underline{x}(x, y, z)$ & Vector observer position \\
\hline$\underline{y}=\underline{y}(x, y, z)$ & Vector source position from the primary nozzle exit \\
\hline $\bar{\beta}-$ & Off-design parameter \\
\hline$\gamma$ & Ratio of specific heats \\
\hline$\delta$ & Dirac delta function \\
\hline$\epsilon$ & Dissipation rate of turbulent kinetic energy \\
\hline$\underline{\eta}=\underline{\eta}(\xi, \eta, \sigma)$ & Vector between two source locations \\
\hline $\bar{\theta}$ & Dilatation rate or observer angle from nozzle downstream axis \\
\hline$\lambda$ & Parameter equal to $\omega \sin \theta / c_{\infty}$ \\
\hline$\pi$ & Logarithm of the pressure \\
\hline$\pi_{g}^{n}$ & Vector Green's function of the linearized Euler equations \\
\hline$\rho$ & Density \\
\hline$\tau$ & Retarded time \\
\hline$\tau_{s}$ & Turbulent integral time scale \\
\hline$\phi$ & Azimuthal angle \\
\hline$\Psi$ & Observer angle from the nozzle inlet axis \\
\hline$\Omega$ & Specific dissipation rate of turbulent kinetic energy \\
\hline$\omega$ & Radian frequency \\
\hline \multicolumn{2}{|l|}{ Subscript } \\
\hline$j$ & Jet fully expanded quantity \\
\hline$p$ & Property of the primary jet \\
\hline$s$ & Property of the secondary jet, shocks, or turbulence scale \\
\hline$\infty$ & Ambient value \\
\hline
\end{tabular}

\section{Introduction}

Broadband shock-associated noise (BBSAN) is present in supersonic jets when the jet is operating offdesign. This occurs due to the static pressure at the nozzle exit not being equal to the pressure outside the nozzle. Nozzles that operate at the nominal on-design pressure ratio can also create a periodic shock cell structure in the jet plume if the characteristic waves are not canceled by the nozzle walls or the flow separates inside the nozzle. BBSAN occurs in nozzles that are convergent or convergent-divergent and when the flow is over- or under-expanded. The interaction between the large-scale coherent turbulence in the jet shear layer and shock cells is the source of shock-associated noise. BBSAN is observed in the far-field as a 
broad spectral peak and dominates the jet mixing noise levels at large angles to the jet downstream axis. The peak frequency is a function of the jet shock cell spacing, the convection velocity of the jet shear layer turbulence, and observer location. The amplitude of BBSAN depends on the ratio of observer distance to the jet diameter, the polar and azimuthal observer angles, the geometry of the nozzle, the degree of offdesign operation, and to a lesser degree, the stagnation temperature. The degree of off-design operation is represented by the off-design parameter, which is defined by $\beta=\left|M_{j}^{2}-M_{d}^{2}\right|^{1 / 2} . M_{j}$ is the fully-expanded jet Mach number, which depends on the nozzle pressure ratio and the ratio of specific heats, and $M_{d}$ is the nozzle design Mach number, which depends on the nozzle throat to exit area ratio.

Harper-Bourne and Fisher ${ }^{2}$ developed the first prediction method for BBSAN. Their proposition was that BBSAN depends on the nearly coherent interaction between the turbulence in the jet shear layer and the jets nearly periodic shock cell structure. This can be modeled as a series of correlated point sources that radiate either constructively or destructively. Harper-Bourne and Fisher's prediction scheme depends on knowledge of the rate of decay of the turbulence correlation between shocks, as well as the characteristic spectral shape of the radiated noise generated by each interaction. These were obtained using a least squares procedure to match the model with experimental noise measurements. The method is used in the SAE ARP $876^{3}$ prediction method for single stream shock-associated noise from convergent nozzles at supercritical conditions and is included as a prediction module in NASA's Aircraft Noise Prediction Program (ANOPP). ${ }^{4}$

$\mathrm{Tam}^{5}$ developed a method for BBSAN prediction and the basic physical model is described by Tam and Tanna. ${ }^{6}$ Tam argued that the shock cell structure in the jet could be modeled, following the work of Pack, ${ }^{7}$ as a waveguide, where the waves are forced by the pressure imbalance at the jet exit and are confined by the jet shear layer. The simplest model that can be used for the jet is a vortex sheet. The effects of the slow divergence of the jet and the dissipative effects of the turbulence on the shock cells can also be included in the same general framework as shown by Tam et al. ${ }^{8}$ The large-scale turbulence in the jet shear layer is modeled as a random superposition of instability waves supported by the jet mean flow, as described by Tam and Chen. ${ }^{9}$ The interaction between the downstream traveling instability waves and the nearly periodic shock cell structure results in an interference pattern of traveling waves. The phase velocity of these waves can be higher than that of the instability waves alone and gives rise to noise radiation at large angles to the jet downstream axis, including the upstream direction. Since there is a random set of instability waves interacting with the shock cells, the resulting radiation pattern involves broad lobes rather than a sharply directional radiation. Empirical formulas are used to represent the shock cell spacing, the convection velocity of the turbulence, and the spectral width of the measured noise data. The predictions give good agreement with noise measurements in both the jet's near- and far-fields and certain key features of the measured spectra are captured. These include the variation of the frequency of the broadband spectral peak with observer location (the same prediction is provided by the Harper-Bourne and Fisher ${ }^{2}$ model), the narrowing of the width of the noise spectrum as the observer moves towards the jet upstream direction, the presence of multiple lobes in the near-field noise contours, and the presence of secondary spectral peaks at higher frequencies than the main peak. It should be noted that the Harper-Bourne and Fisher ${ }^{2}$ model predicted multiple harmonics of the shock peak with the same amplitude, which is not observed in the experiments. This can be overcome if the shock cell spacing is taken to be non-uniform. More recent versions of ANOPP have incorporated Tam's BBSAN model.

$\mathrm{Tam}^{10}$ modified the model by Tam ${ }^{5}$ to include the capability to predict BBSAN from heated jets up to a moderate off-design parameter. This was accomplished by better approximating the shock cell spacing. Also, a temperature correction factor, $T_{c f}$, was included to correct the over-prediction at all frequencies due to increasing total temperature ratio $(T T R)$. Another important modification was the use of different scaling factors that were dependent on whether the jet was over- or under-expanded.

Morris and Miller ${ }^{11}$ developed a prediction method for BBSAN that uses a RANS CFD solution for the properties of the flow-field. The instantaneous properties were decomposed into the mean field quantities, a shock perturbation, a turbulence perturbation, and a perturbation due to the interaction of the turbulence and shocks, which is the BBSAN. The resulting equations were rearranged to form the operator of the linearized Euler equations for the BBSAN fluctuations on the left hand side with resultant equivalent source terms placed on the right hand side. This forms an acoustic analogy for the BBSAN. The solution for the far-field pressure can be written immediately in terms of the source terms and the vector Green's function of the linearized Euler equations. The vector Green's function was written in terms of the solution of the Green's function for the Helmholtz equation. This approximation was made because the BBSAN sources are located in the shear layer of the jet and radiate predominantly in the sideline and upstream directions. 
Sources are modeled based on dimensional grounds and scale as the shock pressure and turbulent velocity fluctuations, and an integral length scale of turbulence in the jet shear layer. The cross-correlation of the jet turbulence is modeled in a separable form as first suggested by Ribner ${ }^{12}$ and scales with the turbulent kinetic energy. The final closed form model equation is a volume integral over the jet plume and a single integral of the shock cell pressure wavenumber spectrum. All the quantities in the closed form model equation can easily be found based on a steady Reynolds-Averaged Navier-Stokes (SRANS) solution. Simple models relate the SRANS solution to the turbulence quantities.

Dual stream jet SRANS calculations have recently been performed by Abdelhamid and Ganz ${ }^{13}$ to investigate the characteristics of the shock-containing plumes. They showed that the shock cell spacing and strength were predicted correctly over a wide range of operating conditions. SRANS calculations can capture realistic mean flow values for BBSAN prediction. In dualstream jets, there are essentially two sets of BBSAN sources. One that occurs in the outer shear layer that divides the fan stream and the ambient air and another that occurs in the inner shear layer that divides the core and fan streams. Tam et al. ${ }^{14}$ confirmed this by predicting the peak frequencies from dualstream jets where the core stream is subsonic and the fan stream is supersonic and operating off-design. This was achieved by accurately predicting the peak BBSAN frequencies for both sets of shock wave shear layer interactions over a wide range of observer angles.

Tam and Reddy ${ }^{15}$ developed a rectangular jet BBSAN prediction model for aspect ratios less than six and with convergent-divergent geometry. One of the side-walls of the rectangular nozzle has no variation. The model is a modification of the work performed by Tam. ${ }^{5}$ The model is linked to a rectangular nozzle with one straight wall, thus the nozzle always supports a shock cell structure. Further modifications to this model have been made to include the effects of forward flight.

The model of Morris and Miller ${ }^{11}$ was applied to dualstream and rectangular jets by Miller and Morris. ${ }^{16}$ This work demonstrated the first BBSAN predictions of dualstream jets using an acoustic analogy. However, the approximation that the mean flow has negligible effect on the noise is not appropriate for dualstream jets if the dominant shock noise is from the primary stream. This is due to the refraction effects of the secondary stream shear layer. Miller and Morris ${ }^{16}$ also demonstrated the first rectangular nozzle BBSAN predictions based on SRANS solutions. The developed model equation makes no assumption regarding the geometry of the nozzle and is not limited to rectangular or circular geometries.

Many empirical models have been developed for jet mixing noise. There are a limited number of empirical models developed for BBSAN. Unlike the models discussed thus far, these are based on noise measurements in the far-field and typically predict only spectra in one-third octave bands. One such example is the ANOPP module of Stone ${ }^{4}$ designed for singlestream or high bypass dualstream axisymmetric nozzles. It follows a traditional empirical noise modeling strategy of forming the mean square pressure as a product of the acoustic power, directivity, and a spectrum function. This model has been validated extensively against a large experimental database. Another notable empirical model is by Deneuville ${ }^{17}$ that essentially consists of a peak frequency and magnitude at a point and calculates the rise and fall of BBSAN as straight lines.

Kuo et al. ${ }^{18}$ performed experimental studies on various jets operating off-design and at various total temperature ratios. The effects of these variations in temperature were compared to the far-field spectra in an attempt to find scaling laws of BBSAN. A simple empirical model was constructed that fits the spectral shapes across a large range of operating conditions. Like the model of Tam, ${ }^{5}$ the BBSAN spectra are chosen to consist of Gaussian peaks. Recently Viswanathan et al. ${ }^{19}$ measured the saturation of BBSAN with stagnation temperature and separated out the fine-scale mixing noise.

Recently Dahl ${ }^{20}$ has compared many BBSAN model predictions to experiment. These include some of the models discussed in this paper and also includes a brief comparison of the SRANS based model of Morris and Miller ${ }^{1}$ with those of Tam. ${ }^{10}$ Dahl showed that for cold cylindrical jets operating supersonically and off-design the models of Tam, ${ }^{10}$ Morris and Miller, ${ }^{11}$ Stone,${ }^{4}$ and Deneuville ${ }^{17}$ all had good agreement for the peak frequency, peak levels, and directivity angles. When the cylindrical jet is heated the BBSAN models of Tam ${ }^{5}$ and Morris and Miller ${ }^{1}$ correctly predict the magnitude and frequencies in the upstream direction but none of the models accurately predict the magnitude in the downstream direction. However, this is less of a concern due to mixing noise typically being dominant in the downstream quadrant of jet.

In this paper an acoustic analogy is developed that is based on the Euler equations for the prediction of BBSAN. The propagation is addressed by requiring knowledge of the vector Green's functions to the linearized Euler equations as a component of the solution to the acoustic analogy equations. Any adjoint vector Green's function solver for the linearized Euler equations could be used in the solution. In this work the vector Green's function is found numerically by using a locally parallel flow assumption that is similar 
to the approach of Tam and Auriault, ${ }^{21}$ Raizada, ${ }^{22}$ and Khavaran et al. ${ }^{23}$ Predictions using the newly developed BBSAN model are compared with the previously developed model and experimental data from the National Aeronautics and Space Administration (NASA), the Pennsylvania State University (PSU), and the Boeing Company.

In addition to the prediction of BBSAN, the prediction of so-called 'fine-scale' mixing noise is performed using the models of Tam and Auriault ${ }^{24}$ and Morris and Boluriaan. ${ }^{25}$ The fine-scale mixing noise component radiates in all directions from the jet and is overshadowed by the noise from large-scale structures in the downstream direction and partially by the BBSAN in the sideline directions. It occurs at all jet Mach numbers. These fine-scale mixing noise models are simple to implement in the developed computer code RANS Integration for Shock Noise (RISN) due to its modular nature and the similar arguments used in BBSAN models. The fine-scale mixing noise models use the same form of the vector Green's function that are used in the newly developed BBSAN model. The fine-scale mixing noise models are exercised using the same nozzles and operating conditions as the BBSAN models. The combination of fine-scale mixing and BBSAN predictions yields a prediction of the total noise created by off-design supersonic heated jets. However, it should be noted that no model is included for the large-scale mixing noise in the peak noise radiated direction.

The next section describes the development of the new prediction model in detail. Next, details regarding the implementation of the BBSAN model in RISN are given. The determination of various arguments of the mathematical models and the evaluation of the models themselves are then described. Extensive predictions are performed using the developed models using the RISN code and comparisons are made with experimental data. A wide range of design Mach numbers and operating conditions are selected to compare the predictions with experiment. Finally, conclusions are drawn based on the developed BBSAN models and predictions.

\section{Mathematical Model}

Mean flow effects in the previously developed BBSAN model of Morris and Miller ${ }^{1}$ have been neglected, and an approximation of the vector Green's function of the linearized Euler equations was used. This involved using the Green's function of the Helmholtz equation and writing it in terms of the vector Green's function for the linearized Euler equations. A similar modeling strategy has been followed here except it is assumed that the vector Green's function to the actual linearized Euler equations is known explicitly. This model is similar in form to the one previously developed because it consists of a single wavenumber integral in the axial direction of the shock pressure spectrum and a volume integral over the BBSAN sources. It can be shown that this model, when the previous form of the vector Green's functions are inserted, reduces to the model originally developed by Morris and Miller. ${ }^{11}$ The newly formed prediction method has the same advantages as the previous model, but can accurately take into account the mean flow effects that are present due to high speed shear layers and freestream Mach numbers.

A vector Green's function solver for the linearized Euler equations is required to complete the newly developed prediction model. Tam and Auriault ${ }^{24}$ and Karabasov et al. ${ }^{26}$ have developed a three-dimensional adjoint solver for the linearized Euler equations with a general mean flow. However, here a simpler approach is adopted temporarily by the authors that is based on a locally parallel flow approximation. Since the spreading rate of the jet is small, this approach is a reasonable first approximation. At each streamwise location of the jet, field variables are extracted to calculate the Green's function of Lilley's equation. This is performed by solving a single differential equation numerically. Only for very special mean flows can the equation be solved analytically. The resultant Green's function of Lilley's equation is used to calculate the vector Green's function of the linearized Euler equations. This step is performed at each streamwise location for each observer angle and frequency. The methodology of this approach is based on Tam and Auriault, ${ }^{21}$ Raizada, ${ }^{22}$ and Karabasov et al. ${ }^{26}$

The governing equations chosen to form the acoustic analogy are the Euler equations. This is similar to the approach developed by Tam. ${ }^{5}$ Tam's analysis is considerably simplified if the following form of the inviscid compressible equations of motion are used,

$$
\begin{gathered}
\frac{D \pi}{D t}+\frac{\partial v_{i}}{\partial x_{i}}=0 \\
\frac{D v_{i}}{D t}+c^{2} \frac{\partial \pi}{\partial x_{i}}=0
\end{gathered}
$$


where $D$ is the material derivative, $c$ is the local speed of sound, $t$ is time, and $v_{i}$ are the velocity components in the $x_{i}$ directions of a Cartesian coordinate system. $\pi$ is related to the logarithm of the pressure,

$$
\pi=\frac{1}{\gamma} \ln \left(p / p_{\infty}\right)
$$

where $p$ is the pressure, $p_{\infty}$ is the ambient pressure, and $\gamma$ is the ratio of specific heats of an ideal gas.

Following Tam, ${ }^{5}$ the instantaneous flow-field properties are separated into four components. That is,

$$
\left[\begin{array}{l}
\pi \\
v_{i}
\end{array}\right]=\left[\begin{array}{l}
\bar{\pi}+\pi_{s}+\pi_{t}+\pi^{\prime} \\
\bar{v}_{i}+v_{s i}+v_{t i}+v_{i}^{\prime}
\end{array}\right]
$$

where the overbar denotes the long time averaged value, the subscript $s$ denotes the perturbations associated with the shock cell structure, the subscript $t$ denotes the fluctuations associated with the turbulence, and the primes denote the fluctuations generated by the interaction of the turbulence and the shock cell structure. It will be assumed that the shock cell structure satisfies the steady linearized version of Eqns. 1 and 2. In addition, it is assumed that the unsteady linearized version of these equations is also satisfied by the turbulent velocity fluctuations. This is justified if the important components of the turbulence, so far as the broadband shock-associated noise is concerned, are coherent over relatively large axial distances. These components are described well by a linear instability wave model.

Making these assumptions, the inhomogeneous equations for the fluctuations associated with the interaction of the turbulence with the shock cells can be written,

$$
\begin{gathered}
\frac{\partial \pi^{\prime}}{\partial t}+\bar{v}_{j} \frac{\partial \pi^{\prime}}{\partial x_{j}}+\frac{\partial v_{i}^{\prime}}{\partial x_{i}}=\theta \\
\frac{\partial v_{i}^{\prime}}{\partial t}+\bar{v}_{j} \frac{\partial v_{i}^{\prime}}{\partial x_{j}}+v_{j}^{\prime} \frac{\partial \bar{v}_{i}}{\partial x_{j}}+\bar{c}^{2} \frac{\partial \pi^{\prime}}{\partial x_{i}}=f_{i}^{v}+f_{i}^{a}
\end{gathered}
$$

The terms on the left hand side of Eqns. 5 and 6 are the linearized Euler equations in terms of the perturbation quantities $\pi^{\prime}$ and $v_{i}^{\prime}$. The terms that appear on the right hand sides represent the equivalent sources of the BBSAN. They are defined by,

$$
\begin{gathered}
\theta=-v_{s j} \frac{\partial \pi_{t}}{\partial x_{j}}-v_{t j} \frac{\partial \pi_{s}}{\partial x_{j}} \\
f_{i}^{v}=-v_{s j} \frac{\partial v_{t i}}{\partial x_{j}}-v_{t j} \frac{\partial v_{s i}}{\partial x_{j}} \\
f_{i}^{a}=-c_{s}^{2} \frac{\partial \pi_{t}}{\partial x_{i}}-c_{t}^{2} \frac{\partial \pi_{s}}{\partial x_{i}}
\end{gathered}
$$

where $\theta$ is a dilatation rate generated by the interaction between the pressure gradients and the turbulent velocity perturbations and the shock cells. $f_{i}^{v}$ is the unsteady force per unit volume associated with interactions between the turbulent velocity fluctuations and the velocity perturbations associated with the shock cells. Finally, $f_{i}^{a}$ is the unsteady force per unit volume related to the interaction of fluctuations in the sound speed (or temperature), caused by the turbulence and the shock cells, and the associated pressure gradients. In traditional approaches to turbulence mixing noise models these equivalent sources have been treated separately and, for the moment, the same assumption will be made here. The solution to Eqns. 5 and 6, can be written in terms of the vector Green's function that satisfies the equations,

$$
\begin{gathered}
\frac{\partial \pi_{g}^{n}}{\partial t}+\bar{v}_{j} \frac{\partial \pi_{g}^{n}}{\partial x_{j}}+\frac{\partial v_{g i}^{n}}{\partial x_{i}}=\delta(\underline{x}-\underline{y}) \delta(t-\tau) \delta_{0 n} \\
\frac{\partial v_{g i}^{n}}{\partial t}+\bar{v}_{j} \frac{\partial v_{g i}^{n}}{\partial x_{j}}+v_{g j}^{n} \frac{\partial \bar{v}_{i}}{\partial x_{j}}+\bar{c}^{2} \frac{\partial \pi_{g}^{n}}{\partial x_{i}}=\delta(\underline{x}-\underline{y}) \delta(t-\tau) \delta_{i n}
\end{gathered}
$$

where $\pi_{g}^{n}=\pi_{g}^{n}(\underline{x}, \underline{y}, t-\tau)$ and $v_{g i}^{n}=v_{g i}^{n}(\underline{x}, \underline{y}, t-\tau)$ are the components of the vector Green's function, $\underline{x}$ denotes the observer position, $\underline{y}$ denotes the source location, $\delta()$ is the Dirac delta function, and $\tau$ is the 
source emission time. $\delta_{i j}$ is the Kronecker delta function. For small perturbation pressures, $\pi^{\prime} \simeq p^{\prime} / \gamma p_{\infty}=$ $p^{\prime} / \rho_{\infty} c_{\infty}^{2}$. Then the solution for the far-field pressure $p^{\prime}(\underline{x}, t)$ can be written,

$$
\begin{aligned}
p^{\prime}(\underline{x}, t)= & \rho_{\infty} c_{\infty}^{2} \iint_{-\infty}^{\infty} \iint_{-\infty}^{\infty} \pi_{g}^{0}(\underline{x}, \underline{y}, t-\tau) \theta(\underline{y}, \tau) \\
& +\sum_{n=1}^{3} \pi_{g}^{n}(\underline{x}, \underline{y}, t-\tau)\left[f_{n}^{v}+f_{n}^{a}\right](\underline{y}, \tau) d \tau d \underline{y}
\end{aligned}
$$

The periodic Green's function is also introduced, given by,

$$
\begin{gathered}
\pi_{g}^{n}(\underline{x}, \underline{y}, \omega)=\frac{1}{2 \pi} \int_{-\infty}^{\infty} \pi_{g}^{n}(\underline{x}, \underline{y}, t-\tau) \exp [-i \omega(t-\tau)] d t \\
\pi_{g}^{n}(\underline{x}, \underline{y}, t-\tau)=\int_{-\infty}^{\infty} \pi_{g}^{n}(\underline{x}, \underline{y}, \omega) \exp [i \omega(t-\tau)] d \omega
\end{gathered}
$$

From this point, only the source term associated with the velocity perturbations will be considered. It is expected that the scaling of the other source terms would be similar. The exception would be the source term associated with the temperature fluctuations. However, the importance of this term still remains the subject of debate in the prediction of turbulent mixing noise in heated jets. So, for the moment, this term will not be considered further. Then, the pressure is given by,

$$
p^{\prime}(\underline{x}, t)=\frac{\rho_{\infty} c_{\infty}^{2}}{2 \pi} \int_{-\infty}^{\infty} \cdots \int_{-\infty}^{\infty} \sum_{n=1}^{3} \pi_{g}^{n}(\underline{x}, \underline{y}, \omega) f_{n}^{v}(\underline{y}, \tau) d \omega d \tau d \underline{y}
$$

Now the autocorrelation of the pressure can be formed. It is given by,

$$
\begin{aligned}
& \overline{p^{\prime}(\underline{x}, t) p^{\prime}\left(\underline{x}, t+\tau^{*}\right)}=\frac{\rho_{\infty}^{2} c_{\infty}^{4}}{(2 \pi)^{2}} \int_{-\infty}^{\infty} \ldots \int_{-\infty}^{\infty} \sum_{n=1}^{3} \sum_{m=1}^{3} \pi_{g}^{n}\left(\underline{x}, \underline{y}, \omega_{1}\right) \pi_{g}^{m}\left(\underline{x}, \underline{z}, \omega_{2}\right) \\
& \times \overline{f_{n}^{v}\left(\underline{y}, \tau_{1}\right) f_{m}^{v}\left(\underline{z}, \tau_{2}\right)} \exp \left[-i \omega_{1}\left(t-\tau_{1}\right)-i \omega_{2}\left(t-\tau_{2}\right)-i \omega \tau^{*}\right] d \omega_{1} d \omega_{2} d \tau_{1} d \tau_{2} d \underline{y} d \underline{z}
\end{aligned}
$$

The spectral density is given by the Fourier transform of the autocorrelation of the pressure,

$$
S(\underline{x}, \omega)=\int_{-\infty}^{\infty} \overline{p^{\prime}(\underline{x}, t) p^{\prime}\left(\underline{x}, t+\tau^{*}\right)} \exp \left(i \omega \tau^{*}\right) d \tau^{*}
$$

The integration with respect to $\tau^{*}$ can be performed immediately.

Before proceeding it is necessary to examine the form of the two-point cross-correlation of $f_{n}^{v}$. It is dependent on the strength of the shock cells and the turbulent fluctuations and its product is significant in regions where the shocks and expansions intersect with the turbulent shear layer. That is, if there is no turbulence present or pressure perturbation due to shock cells, then the term is small. Furthermore, the amplitude of $f_{n}^{v}$ is proportional to the shock cell pressure perturbations and the turbulent velocity fluctuations. If it is assumed that the two-point cross correlation function of the BBSAN source term can be written in terms of separation distance $\underline{\eta}$ and time delay $\tau$, then we can write,

$$
S(\underline{\mathrm{x}}, \omega)=\rho_{\infty}^{2} c_{\infty}^{4} \int_{-\infty}^{\infty} \ldots \int_{-\infty}^{\infty} \sum_{n=1}^{3} \sum_{m=1}^{3} \pi_{g}^{n}(\underline{\mathrm{x}}, \underline{\mathrm{y}},-\omega) \pi_{g}^{m}(\underline{\mathrm{x}}, \underline{\mathrm{y}}+\underline{\eta}, \omega) R_{n m}^{v}(\underline{\mathrm{y}}, \underline{\eta}, \tau) \exp [i \omega \tau] d \tau d \underline{\eta} d \underline{\mathrm{y}}
$$

where $R_{n m}^{v}$ is the two-point cross-correlation of the equivalent source term $f_{n}^{v}$,

$$
R_{n m}^{v}(\underline{\mathrm{y}}, \underline{\eta}, \tau)=\overline{f_{n}^{v}(\mathrm{y}, t) f_{m}^{v}(\underline{\mathrm{y}}+\underline{\eta}, t+\tau)}
$$


and

$$
f_{n}^{v}(\underline{\mathrm{y}}, t)=-v_{s j} \frac{\partial v_{t n}}{\partial x_{j}}-v_{t j} \frac{\partial v_{s n}}{\partial x_{j}}
$$

The SRANS solution does not provide any information about the form of $f_{n}^{v}$. A model for $R_{n m}^{v}$ is constructed as,

$$
R_{n m}^{v}(\underline{\mathrm{y}}, \underline{\eta}, \tau)=\frac{1}{\rho_{\infty}^{2} c_{\infty}^{2} l^{2}} p_{s}(\mathrm{y}) p_{s}(\underline{\mathrm{y}}+\underline{\eta}) R_{m n}(\underline{y}, \underline{\eta}, \tau)
$$

where $R_{n m}$ is the two-point cross correlation function of the turbulent velocity fluctuations in the $n$ and $m$ directions. Over the distance where the spatial correlation is significant we also continue to assume that,

$$
\pi_{g}^{m}(\underline{x}, \underline{y}+\underline{\eta},-\omega)=\pi_{g}^{m}(\underline{x}, \underline{\mathrm{y}}, \omega) \exp \left[-i \frac{\omega}{c_{\infty}} \underline{\underline{x}} \cdot \underline{\eta}\right]
$$

as argued by Tam and Auriault. ${ }^{21}$ From the definition of $\pi_{g}^{m}$,

$$
\pi_{g}^{m *}(\underline{x}, \underline{y}, \omega)=\pi_{g}^{m}(\underline{x}, \underline{y},-\omega)
$$

where the superscript $*$ represents the complex conjugate. Now,

$$
\begin{aligned}
S(\underline{\mathrm{x}}, \omega)=c_{\infty}^{2} \int_{-\infty}^{\infty} \ldots \int_{-\infty}^{\infty} \sum_{n=1}^{3} & \sum_{m=1}^{3} \pi_{g}^{n *}(\underline{\mathrm{x}}, \underline{\mathrm{y}}, \omega) \pi_{g}^{m}(\underline{\mathrm{x}}, \underline{\mathrm{y}}, \omega) \frac{p_{s}(\underline{\mathrm{y}}) p_{s}(\underline{\mathrm{y}}+\underline{\eta})}{l^{2}} \\
& \times R_{n m}(\underline{\mathrm{y}}, \underline{\eta}, \tau) \exp \left[i \omega \tau-i \frac{\omega}{c_{\infty}} \underline{\underline{x}} \cdot \underline{\eta}\right] d \tau d \underline{\eta} d \underline{\mathrm{y}}
\end{aligned}
$$

One of the shock cell pressure distributions can be represented in terms of its axial wavenumber spectrum,

$$
\tilde{p}_{s}\left(k_{1}, y_{2}, y_{3}\right)=\int_{-\infty}^{\infty} p_{s}(\mathrm{y}) \exp \left[-i k_{1} y_{1}\right] d y_{1}
$$

and the corresponding inverse Fourier transform is,

$$
p_{s}(\mathrm{y})=\frac{1}{2 \pi} \int_{-\infty}^{\infty} \tilde{p}_{s}\left(k_{1}, y_{2}, y_{3}\right) \exp \left[i k_{1} y_{1}\right] d k_{1}
$$

Then,

$$
\begin{aligned}
S(\underline{\mathrm{x}}, \omega)=\frac{c_{\infty}^{2}}{2 \pi} \int_{-\infty}^{\infty} & \ldots \int_{-\infty}^{\infty} \sum_{n=1}^{3} \sum_{m=1}^{3} \pi_{g}^{n *}(\underline{\mathrm{x}}, \underline{\mathrm{y}}, \omega) \pi_{g}^{m}(\underline{\mathrm{x}}, \underline{\mathrm{y}}, \omega) \frac{p_{s}(\underline{\mathrm{y}}) \tilde{p}_{s}\left(k_{1}, y_{2}+\eta, y_{3}+\zeta\right)}{l^{2}} \\
& \times R_{n m}(\underline{\mathrm{y}}, \underline{\eta}, \tau) \exp \left[-i k_{1}\left(y_{1}+\xi\right)\right] \exp \left[-i \frac{\omega}{c_{\infty}} \frac{\underline{x}}{x} \cdot \underline{\eta}+i \omega \tau\right] d k_{1} d \tau d \underline{\eta} d \underline{\mathrm{y}}
\end{aligned}
$$

Now, we introduce the wavenumber frequency spectrum of the turbulent velocity correlation. That is,

and

$$
S_{n m}\left(\underline{\mathrm{y}}, \underline{k}^{*}, \omega^{*}\right)=\int_{-\infty}^{\infty} \ldots \int_{-\infty}^{\infty} R_{m n}(\underline{\mathrm{y}}, \underline{\eta}, \tau) \exp \left[i\left(\omega^{*} \tau-\underline{k}^{*} \cdot \underline{\eta}\right)\right] d \underline{\eta} d \tau
$$

$$
R_{n m}(\underline{y}, \underline{\eta}, \tau)=\frac{1}{16 \pi^{4}} \int_{-\infty}^{\infty} \ldots \int_{-\infty}^{\infty} S_{n m}\left(\underline{\mathrm{y}}, \underline{k}^{*}, \omega^{*}\right) \exp \left[-i\left(\omega^{*} \tau-\underline{k}^{*} \cdot \underline{\eta}\right)\right] d \omega^{*} d \underline{k}^{*}
$$

Then,

$$
\begin{array}{r}
S(\underline{\mathrm{x}}, \omega)=\frac{c_{\infty}^{2}}{32 \pi^{5}} \int_{-\infty}^{\infty} \ldots \int_{-\infty}^{\infty} \sum_{n=1}^{3} \sum_{m=1}^{3} \pi_{g}^{n *}(\underline{\mathrm{x}}, \underline{\mathrm{y}}, \omega) \pi_{g}^{m}(\underline{\mathrm{x}}, \underline{\mathrm{y}}, \omega) \\
\times \frac{p_{s}(\underline{\mathrm{y}}) \tilde{p}_{s}\left(k_{1}, y_{2}+\eta, y_{3}+\zeta\right)}{l^{2}} \exp \left[-i k_{1}\left(y_{1}+\xi\right)\right] S_{n m}\left(\underline{\mathrm{y}}, \underline{k}^{*}, \omega^{*}\right) \\
\times \exp \left[-i \omega^{*}+i \underline{k}^{*} \cdot \underline{\eta}+i \omega-i \frac{\omega \underline{x}}{c_{\infty} x} \cdot \eta\right] d \omega^{*} d k^{*} d k_{1} d \underline{\eta} d \underline{\mathrm{y}}
\end{array}
$$


In Morris and Miller ${ }^{1}$ it was assumed that the shock cell structure was independent of the cross-stream distance over the distance where $R_{n m}$ remained significant. From an examination of the wavenumber spectrum as a function of $r$, this appears to be a reasonable assumption. Obviously, in the azimuthal direction for axisymmetric jets this also is true. So, we can replace $\tilde{p}_{s}\left(k_{1}, y_{2}+\eta, y_{3}+\zeta\right)$ by $\tilde{p}_{s}\left(k_{1}, y_{2}, y_{3}\right)$ as before. Then,

$$
\begin{aligned}
S(\underline{\mathrm{x}}, \omega)= & \frac{c_{\infty}^{2}}{16 \pi^{4}} \int_{-\infty}^{\infty} \ldots \int_{-\infty}^{\infty} \sum_{n=1}^{3} \sum_{m=1}^{3} \pi_{g}^{n *}(\underline{\mathrm{x}}, \underline{\mathrm{y}}, \omega) \pi_{g}^{m}(\underline{\mathrm{x}}, \underline{\mathrm{y}}, \omega) \exp \left[-i k_{1}\left(y_{1}+\xi\right)\right] \\
& \times \frac{p_{s}(\underline{\mathrm{y}}) \tilde{p}_{s}\left(k_{1}, y_{2}, y_{3}\right)}{l^{2}} S_{n m}\left(\underline{\mathrm{y}}, \underline{k}^{*}, \omega\right) \exp \left[i\left(\underline{k}^{*}-\frac{\omega \underline{x}}{c_{\infty} x}\right) \cdot \underline{\eta}\right] d \underline{k}^{*} d k_{1} d \underline{\eta} d \underline{\mathrm{y}}
\end{aligned}
$$

Use of the integration identity,

$$
\int_{-\infty}^{\infty} \exp \left[-i\left(\omega^{*}-\omega\right) \tau\right] d \tau=2 \pi \delta\left(\omega^{*}-\omega\right)
$$

permits the integration with respect to $\tau$ and $\omega^{*}$ to be performed. Thus,

$$
\begin{aligned}
S(\underline{\mathrm{x}}, \omega)= & \frac{c_{\infty}^{2}}{2 \pi} \int_{-\infty}^{\infty} \ldots \int_{-\infty}^{\infty} \sum_{n=1}^{3} \sum_{m=1}^{3} \pi_{g}^{n *}(\underline{\mathrm{x}}, \mathrm{y}, \omega) \pi_{g}^{m}(\underline{\mathrm{x}}, \mathrm{y}, \omega) \exp \left[-i k_{1} y_{1}\right] \\
& \times \frac{p_{s}(\underline{\mathrm{y}}) \tilde{p}_{s}\left(k_{1}, y_{2}, y_{3}\right)}{l^{2}} S_{n m}\left(\underline{\mathrm{y}}, \frac{\omega x_{1}}{c_{\infty} x}+k_{1}, \frac{\omega x_{2}}{c_{\infty} x}, \frac{\omega x_{3}}{c_{\infty} x}\right) d k_{1} d \underline{y}
\end{aligned}
$$

Now, consider the form of the two-point cross-correlation of the velocity fluctuations. For simplicity it will be assumed that they all have the same shape, but the amplitude will vary depending on the component. This is consistent with the approach used by Karabasov et al. ${ }^{27}$ for the fourth-order cross-correlations. It can be expressed as,

$$
R_{n m}(\underline{y}, \underline{\eta}, \tau)=a_{n m} K R(\underline{\eta}, \tau)
$$

where $K$ is the turbulent kinetic energy and $a_{n m}$ reflects the relative amplitudes of the different components of the second-order cross-correlation. The form chosen for $R(\underline{\eta}, \tau)$ is the same as used before by Morris and Miller. ${ }^{1}$ That is,

$$
R(\underline{\eta}, \tau)=\exp \left[-|\tau| / \tau_{s}\right] \exp \left[-(\xi-\omega \tau)^{2} / l^{2}\right] \exp \left[-\left(\eta^{2}+\zeta^{2}\right) / l_{\perp}^{2}\right]
$$

where it is assumed that the convection velocity is approximated well by the local mean velocity in regions of large values of $K$. From the definition of $S_{n m}(\underline{y}, \underline{k}, \omega)$ given by Eqn. 28 it can be shown that,

$$
S_{n m}(\underline{y}, \underline{k}, \omega)=2 \pi^{3 / 2} a_{n m} K l l_{\perp}^{2} \tau_{s} \frac{\exp \left[-\left(k_{1}^{2} l^{2}+\left(k_{2}^{2}+k_{3}^{2}\right) l_{\perp}^{2}\right) / 4\right]}{1+\left(\omega-k_{1} u\right)^{2} \tau_{s}^{2}}
$$

Substitution of Eqn. 36 into the form of $S_{n m}\left(\mathrm{y}, \omega x_{1} /\left(c_{\infty} x\right)+k_{1}, \omega x_{2} /\left(c_{\infty} x\right), \omega x_{3} /\left(c_{\infty} x\right)\right)$ with $\underline{x}=x(\cos \theta, \sin \theta \cos \phi, \sin \theta \sin \phi)$ yields,

$$
\begin{aligned}
S_{n m}\left(\mathrm{y}, \frac{\omega x_{1}}{c_{\infty} x}+\right. & \left.k_{1}, \frac{\omega x_{2}}{c_{\infty} x}, \frac{\omega x_{3}}{c_{\infty} x}\right)=\frac{2 \pi^{3 / 2} a_{n m} K l l_{\perp}^{2} \tau_{s}}{\left[1+\left(1-M_{c} \cos \theta-u k_{1} / \omega\right)^{2} \omega^{2} \tau_{s}^{2}\right]} \\
& \times \exp \left[-l^{2}\left(k_{1}+\omega \cos \theta / c_{\infty}\right)^{2} / 4-\omega^{2} l_{\perp}^{2} \sin ^{2} \theta /\left(4 c_{\infty}\right)\right]
\end{aligned}
$$

Substitution of Eqn. 37 into Eqn. 33 and simplification yields,

$$
\begin{aligned}
S(\underline{\mathrm{x}}, \omega)=\pi^{1 / 2} c_{\infty}^{2} \int_{-\infty}^{\infty} & \ldots \int_{-\infty}^{\infty} \sum_{n=1}^{3} \sum_{m=1}^{3} \pi_{g}^{n *}(\underline{\mathrm{x}}, \underline{\mathrm{y}}, \omega) \pi_{g}^{m}(\underline{\mathrm{x}}, \underline{\mathrm{y}}, \omega) p_{s}(\underline{\mathrm{y}}) \tilde{p}_{s}\left(k_{1}, y_{2}, y_{3}\right) \exp \left[i k_{1} y_{1}\right] \\
& \times \frac{a_{n m} K l_{\perp}^{2} \tau_{s}}{l} \frac{\exp \left[-l^{2}\left(k_{1}-\omega \cos \theta / c_{\infty}\right)^{2} / 4-\omega^{2} l_{\perp}^{2} \sin ^{2} \theta /\left(4 c_{\infty}^{2}\right)\right]}{1+\left(1-M_{c} \cos \theta-u k_{1} / \omega\right)^{2} \omega^{2} \tau_{s}^{2}} d k_{1} d \underline{y}
\end{aligned}
$$

Equation 38 represents an acoustic analogy based on the Euler equations that uses the vector Green's function of the linearized Euler equations to predict BBSAN. Appendix A shows that this expression reduces 
to the result by Morris and Miller ${ }^{1}$ when the vector Green's function based on the Helmholtz equation and an isotropic source model are used.

Evaluation of Eqn. 38 requires knowledge of the vector Green's function of the linearized Euler equations. An adjoint approach is followed here which shortens calculation time greatly. This approach is based on the work of Tam and Auriault ${ }^{21}$ and Raizada. ${ }^{22}$ Assuming a locally parallel mean flow, $q=q(y, z)$, where $q$ represents the field-variables, the adjoint Green's function can be written,

$$
g_{a}(\underline{x}, \underline{y}, \omega)=\frac{\exp \left[-i \omega\left(x_{1} \cos \theta-R\right) / c_{\infty}\right]}{8 \pi^{2} c_{\infty}^{2} R \omega} \sum_{m=0}^{\infty} f_{m}(r) \cos (m \phi)
$$

where $m$ is an azimuthal mode number. Equation 39 is valid inside the jet $\left(r<R_{o}\right)$. There is no concern about solutions outside the jet as sources of BBSAN do not exist in that region. The quantity $f_{m}$ is found for each mode $m$ by solving the following differential equation in the region $0<r<R_{o}$,

$$
\begin{aligned}
& \frac{\partial^{2} f_{m}}{\partial r^{2}}+\left[\frac{-4 \cos \theta \frac{d \bar{u}}{d r} / c_{\infty}}{1-\bar{u} \cos \theta / c_{\infty}}-\frac{1}{\bar{\rho}} \frac{d \bar{\rho}}{d r}+\frac{1}{r}\right] \frac{\partial f_{m}}{\partial r} \\
& +\left[\frac{\omega^{2}\left(1-\frac{u \cos \theta}{c_{\infty}}\right)}{\gamma p / \rho}+\frac{3\left(\frac{1}{\rho} \frac{\partial \rho}{\partial r} \frac{\partial u}{\partial r}-\frac{\partial^{2} u}{\partial r^{2}}\right) \frac{\cos \theta}{c_{\infty}}}{1-u \cos \theta / c_{\infty}}-\frac{m^{2}}{r^{2}}-\frac{\omega^{2} \cos ^{2} \theta}{c_{\infty}^{2}}\right] f_{m}=0
\end{aligned}
$$

As shown by Tam and Auriault, ${ }^{21}$ the solution near the centerline $(r \simeq \epsilon)$ is $f_{m}=a_{m} r^{m}$. Using this expression, the boundary conditions at the centerline of the jet are specified and a spatial marching problem in the radial direction can be formed at each streamwise location. The details of the marching methods used are discussed in the next section. For each radial mode $m, f_{m}$ needs to be scaled so that the inner-solution matches the outer-solution of $g_{a}$ at $r=R_{o}$. Raizada ${ }^{22}$ showed that a scaling factor can be used for this purpose,

$$
a_{m}=\frac{(-i)^{m} \epsilon_{m} \lambda\left(J_{m}\left(\lambda R_{o}\right) H_{m}^{(1)^{\prime}}\left(\lambda R_{o}\right)-J_{m}^{\prime}\left(\lambda R_{o}\right) H_{m}^{(1)}\right)}{f_{m}\left(R_{o}\right) H_{m}^{(1)}\left(\lambda R_{o}\right) f_{m}^{\prime}\left(R_{o}\right)-H_{m}^{(1)}\left(\lambda R_{o}\right) \lambda f_{m}\left(R_{o}\right)}
$$

where $\epsilon_{m}$ is unity for $m=1$ and is two for all other values of $m, \lambda=\omega \sin \theta / c_{\infty}$, and the primes denote a derivative of the Hankel function or $f_{m}$ with respect to their arguments. The vector Green's function of the linearized Euler equations is related to the Green's function of Lilley's equation by,

$$
\begin{gathered}
\pi_{g}^{0}=\omega^{2} g-2 i u \omega \frac{\partial g}{\partial y_{x}}-u \frac{\partial^{2} g}{\partial y_{x}^{2}} \\
\pi_{g}^{1}(\underline{x}, \underline{y}, \omega)=-\left(i \omega+u \frac{\partial}{\partial y_{x}}\right) \frac{\partial}{\partial y_{x}} g(\underline{\mathrm{x}}, \underline{\mathrm{y}}, \omega) \\
\pi_{g}^{2}(\underline{x}, \underline{y}, \omega)=-\left\{3 \frac{\partial u}{\partial y_{r}} \frac{\partial}{\partial y_{x}}+\left(i \omega+u \frac{\partial}{\partial y_{x}}\right) \frac{\partial}{\partial y_{r}}\right\} g(\underline{\mathrm{x}}, \underline{\mathrm{y}}, \omega) \\
\pi_{g}^{3}(\underline{x}, \underline{y}, \omega)=-\left(i \omega+u \frac{\partial}{\partial y_{x}}\right) \frac{1}{y_{r}} \frac{\partial}{y_{\theta}} g(\underline{\mathrm{x}}, \underline{\mathrm{y}}, \omega)
\end{gathered}
$$

where $\underline{y}=\left(y_{r}, y_{\theta}, y_{x}\right)$ denotes the source position in cylindrical polar coordinates. The reciprocal relation of $g=\bar{g}_{a}$ holds true for this formulation of the adjoint Green's function. Equations 42 through 45 require various terms involving derivatives of the Green's function to Lilley's equation. These expressions are found analytically based on the numerical calculation of $g$. The various derivatives of $g$ used in Eqns. 42 through 45 with respect to the various spatial directions are,

$$
\begin{aligned}
\frac{\partial g}{\partial x} & =\frac{-i \cos \theta}{8 \pi^{2} c_{\infty}^{3} R} \exp \left[-i \omega(x \cos \theta-R) / c_{\infty}\right] \sum_{m=0}^{\infty} f_{m}(r) \cos (m \phi) \\
\frac{\partial^{2} g}{\partial x^{2}} & =\frac{-\omega \cos ^{2} \theta}{8 \pi^{2} c_{\infty}^{4} R} \exp \left[-i \omega(x \cos \theta-R) / c_{\infty}\right] \sum_{m=0}^{\infty} f_{m}(r) \cos (m \phi)
\end{aligned}
$$




$$
\begin{gathered}
\frac{\partial g}{\partial \phi}=\frac{-m}{8 \pi^{2} \omega c_{\infty}^{2} R} \exp \left[-i \omega(x \cos \theta-R) / c_{\infty}\right] \sum_{m=0}^{\infty} f_{m}(r) \sin (m \phi) \\
\frac{\partial}{\partial x}\left(\frac{1}{r} \frac{\partial g}{\partial \phi}\right)=\frac{i m \cos \theta}{8 r \pi^{2} c_{\infty}^{3} R} \exp \left[-i \omega(x \cos \theta-R) / c_{\infty}\right] \sum_{m=0}^{\infty} f_{m}(r) \sin (m \phi)
\end{gathered}
$$

Equations 46 through 49 are used with Eqns. 42 through 45 to find the vector Green's function based on Lilley's equation at various streamwise locations. Equations 42 through 45 and corresponding complex conjugates can be used in Eqn. 38.

\section{Implementation}

The mathematical models developed in the previous section are implemented in the computer program RANS Integration for Shock Noise (RISN). RISN is a collection of jet noise prediction models that are based on various acoustic analogies and are being developed at NASA Langley Research Center (LaRC). The various prediction methodologies allow total noise predictions based on a summation of jet noise components from axisymmetric or three dimensional jets that are heated, off-design, and compressible. Acoustic analogies are developed for each noise source (eg: BBSAN, fine-scale, large-scale, screech, etc.) and the sources and propagation are treated separately.

The implementation of the model in the absence of propagation effects is described fully in Morris and Miller. ${ }^{1}$ The model developed in this paper is implemented using the same strategy but with an additional subroutine to calculate the vector Green's functions. The model Eqn. 38 is evaluated for each observer position $\underline{x}$. This involves an integration over the sources of BBSAN in the jet plume. That is, where the turbulence is interacting with the oblique shock waves in the shear layer. The range of integration in the streamwise direction (for single stream jets) is generally taken to extend from the nozzle exit to the end of the potential core and in the cross-stream direction from the centerline axis through twice the nozzle diameter. The integral associated with the axial wavenumber $k_{1}$, is performed by using the trapezoidal rule. Only peaks of $\tilde{p}_{s}$ are included in the numerical integration as small values of $\tilde{p}_{s}$ have negligible contribution to the magnitude of BBSAN. This is accomplished by integrating from the main peak of $\tilde{p}_{s}$ to typically half the range of $\tilde{p}_{s}$ over both negative and positive wavenumbers.

The Fourier transform of the shock cell pressure is found by extracting the static pressure along a line from the nozzle lip through the termination of each oblique shock wave. Typically this line extends from the nozzle lip to a downstream location near the jet centerline axis past the potential core where the shock pressure is near ambient value. These pressures are transformed to a shock pressure, $p_{s}$, by subtracting the ambient pressure, $p_{\infty}$. This data is mirrored about $x=0$ and a Hanning window is applied. A discrete Fourier transform is performed and the Hanning window correction factor of eight thirds is applied to the amplitude. This process yields $\tilde{p}_{s}$.

The newly developed prediction model and other associated models in RISN require a knowledge of a mean flow. Mean flows are determined from solutions of the Steady Reynolds-Averaged Navier-Stokes Equations (SRANS). The SRANS solutions are found using the NPARC Alliance Wind-US 2.0 solver. Wind-US was developed by the NPARC Alliance which is a partnership between NASA Glenn Research Center (GRC), the U. S. Air Force Arnold Engineering Development Center, and additional contributors. Solutions from Wind-US are found using the default options of the CFD solver. The SRANS equations are closed by the Menter ${ }^{28}$ shear stress transport (SST) turbulence model.

The computational grids for the SRANS solutions are constructed with Gridgen. The nozzle geometries used in the corresponding experiments are imported from Computer Aided Design (CAD) files from which the nozzles were manufactured. A computational domain for the jet plume is constructed downstream of the nozzle exit. The inside and outside walls of the nozzles, along with the nozzle lip thickness, are all accurately incorporated in the computational grid. The computational domains are structured and contain a single block representing the inside of the nozzle, a single block representing the outside of the nozzle upstream from the nozzle lip, and finally a single block that represents the plume region of the jet. An additional nozzle interior block is used for the dual stream jets. The plume region extends 100 nozzle diameters downstream from the nozzle exit and fifty nozzle diameters in the radial direction from the jet centerline. Based on the largest fully expanded Mach number, total temperature ratio, and $y^{+}=1$, the distance from the wall to the first grid point is set at the nozzle exit. This distance is maintained through the entire internal region of 
the nozzle. This spacing allows the turbulent boundary layer at the nozzle wall to be resolved. The distance from the nozzle centerline to the first internal gridpoint in the radial direction is ten times the value of the distance from the first grid point to the wall at the nozzle exit. A complete description of the computational grids and methods for finding Wind-US 2.0 SRANS solutions with the Menter ${ }^{28}$ SST model can be found in Miller and Veltin. ${ }^{29}$

The developed model equations and the adjoint vector Green's function solver both make use of the SRANS solutions. Specifically, the adjoint vector Green's function solver requires radial profiles of the mean flow solution at each streamwise location. Unfortunately, the mean flow computational grid that is in WindUS is too coarse to march in the $r$ direction using a Runge-Kutta scheme when solving Eqn. 40. The adjoint vector Green's function solver first examines the mean flow solution at the current streamwise location and interpolates the values of the mean flow onto a finer grid. The spacing of the grid is set at $1 \times 10^{-5}$ meters. The range of the interpolated region extends from $0.0001 m<r<r_{\max }$. Typically $r_{\max }$ is equal to the diameter of the nozzle. Equation 40 is evaluated at each streamwise location by marching from $r=0.0001$ $\mathrm{m}$ in the positive $r$ direction using fourth-order accurate Runge-Kutta integration. The initial conditions for Eqn. 40 are $f_{m}=a_{m} r^{m}$ and $d f_{m} / d r=a_{m} m r^{m-1}$ for each mode. The values of $a_{m}$ are found to match the inner and outer solutions of undisturbed incoming waves, and are found from Eqn. 41.

Equation 39 uses the values of $f_{m}$ at each streamwise location $x$ and frequency. Values of $f_{m}$ are never stored but summed when calculating $g_{a}(\underline{x}, y, \omega)($ Eqn. 39) as the index $m$ is increased. The maximum value of $m$ used in these calculations is set at $7 \overline{7}$. Higher values do not increase the accuracy of the numerical calculation. After the summation of $f_{m}(r) \cos (m \phi)$ is complete, it is multiplied by the prefactor shown in Eqn. 39. The adjoint Green's function of the linearized Euler equations for a locally parallel mean flow is now known as $g_{a}$. Recall that the vector Green's function used in the developed acoustic analogy is not based on a shear layer with a parallel mean flow but with an arbitrary mean flow. Thus, it is necessary to relate the form of $g_{a}$ to that of the vector Green's function used in Eqn. 38. First, the derivatives of $g_{a}$ are calculated based on Eqns. 46 through 49. Note that these equations involve the same summation involved in Eqn. 39 but not for Eqns. 48 and 49. During the summation involving $f_{m} \cos (m \phi)$, the summation of $f_{m}(r) \sin (m \phi)$ is also performed for sub-sequential calculation of Eqn. 48 and 49. As before, these summations are performed over modes zero through 77 , and then multiplied by their respective prefactors.

The values of $g_{a}$ and associated derivatives are used as arguments in Eqns. 42 through 45 which in turn, are arguments for Eqn. 38. Recall that the SRANS solution and grid used for integration of Eqn. 38 reside on much coarser computational grids relative to the discrete solution of Eqn. 39. This is due to the need to solve $f_{m}$ accurately at each streamwise location. This fine grid does not necessarily have grid points at locations that correspond to the integration region used in Eqn. 38 in the radial direction. Thus, it is necessary for the adjoint vector Green's function solver to interpolate the values of $g_{a}$ and $\pi_{g}^{n}$ onto the same grid points that are used by Eqn. 38. Linear interpolation is used for this process at each streamwise location. This interpolation is performed by the adjoint vector Green's function solver subroutine and returned as arguments to the subroutine that contains Eqn. 38. That is, the radially varying discrete values of $g_{a}$ and $\pi_{g}^{n}$ are returned to the calling routine as arrays that correspond to the current streamwise location. The adjoint vector Green's function solver is written in a general fashion so that the noise models of Tam and Aurialt ${ }^{24}$ Morris and Boluriaan, ${ }^{25}$ and the newly developed BBSAN model, all use the same subroutine without modification. This is advantageous because the same values of $g_{a}$ and $\pi_{g}^{n}$, based on the same SRANS solution, are returned to each model.

The fine-scale mixing noise models of Tam and Aurialt ${ }^{24}$ and Morris and Boluriaan ${ }^{25}$ have been implemented in the computer program RISN. These models provide expressions for the spectral density due to the fine-scale turbulent mixing and are similar or based on acoustic analogies. These models are simple to implement due in part to the modular design of the RISN code and the large number of arguments that are similar to those of the BBSAN models. Both models consist of spatial integrals that involve mean flow components, turbulence statistics based on the mean flow, and $g_{a}$ or $\pi_{g}^{n}$. In the model of Tam and Auriault ${ }^{24}$ the Green's function is based on the parallel flow assumption and is the same Green's function of Eqn. 39. The model of Morris and Boluriaan ${ }^{25}$ uses the form of the vector Green's function of the linearized Euler equations shown in Eqns. 42 and 43. The vector Green's function of the linearized Euler equations is required for the evaluation of the developed prediction model for BBSAN in Eqn. 38. Thus, it is simple to include these mixing noise models as additional calculations in the RISN code as two additional subroutines.

The BBSAN models contain coefficients that relate the turbulent kinetic energy and dissipation to characteristic turbulence length, velocity, and time scales. The mixing noise models of Tam and Auriault ${ }^{24}$ and 
Morris and Boluriaan ${ }^{25}$ contain similar relations. The same simple models are chosen for $l, l_{\perp}, \tau$, and $u_{c}$ for both mixing-noise prediction methods. However, different coefficients and scaling prefactors are required for each model. The fine-scale mixing noise model of Tam and Auriault ${ }^{24}$ uses a mean flow based on a $K-\epsilon$ model that was specifically designed by Tam and Ganesan ${ }^{30}$ for hot axisymmetric jet flows. The SRANS solutions obtained for the present predictions are based on the Menter ${ }^{28}$ SST turbulence model closure coefficients and the Wind-US 2.0 solver. There is a clear difference between solutions produced by different CFD solvers and turbulence models. An effort has been made to calibrate the coefficients of the model of Tam and Auriault ${ }^{24}$ to correspond to the Menter SST turbulence model. It is acknowledged there is some difference in the mean flows between the two closure schemes. This calibration includes the prefactor scaling coefficient. The same calibration has been performed for the model of Morris and Boluriaan, ${ }^{25}$ which is based on a general CFD SRANS solution such as one produced by Wind-US. The coefficients used in the model of Morris and Boluriaan ${ }^{25}$ are those used in the paper and only the prefactor has been changed.

RISN is able to make noise predictions using either unstructured or structured SRANS solutions. The type of grid, tetrahedral, polyhedral, etc., has no bearing on the prediction for any kind of noise model. This advantage is obtained by interpolating the mean flow and turbulence statistics onto another computational domain (integration region) that is structured and positioned in the jet plume. Inverse distance weighted interpolation using close points is used to find the integration region values. The integration region contains the jet noise sources. This greatly simplifies the integration techniques used in the code as it is independent of the CFD grid. The BBSAN noise models of Eqns. 54 and 38, and the fine-scale turbulent mixing noise models of Morris and Boluriaan ${ }^{25}$ and Tam and Auriault ${ }^{24}$ all use the same schemes to interpolate the CFD solution onto the structured integration region. The integration region is structured and has constant grid spacing in the streamwise and radial directions. It can easily be placed anywhere in the CFD domain and interpolation can occur across blocks of the CFD solution. Grid independence studies have been conducted to ensure that the SRANS solution has converged. Details of these convergence studies are given by Miller and Veltin. ${ }^{31}$ A separate grid independence study is also conducted for each noise model implemented in RISN. The range in the streamwise and radial directions is varied as well as the number of grid points in the streamwise and cross-stream direction. These values are all increased individually for each case until the $S P L$ at the lowest amplitudes calculated vary by less than $0.5 \mathrm{~dB}$. The integration ranges of the BBSAN models are discussed at length in Miller and Morris. ${ }^{29}$ The ranges of the mixing noise integration region varies from case to case. In general, the integration region extends from the nozzle exit to a distance of forty nozzles downstream $(0<x<40 D)$ and in the radial direction from the centerline axis to a distance of ten nozzle diameters $(0<r<10 D)$.

\section{Results}

This section presents predictions using the BBSAN and fine-scale mixing noise models. First, a description of the nozzles is given. The operating conditions for the nozzles are then shown. The CFD solutions of these nozzles operating over a range of conditions is discussed. Finally, the predictions based on the noise models are compared with experimental data.

A number of nozzle geometries and operating conditions have been selected. The convergent nozzle SMC000, shown in Fig. 1, and exit diameter of 0.0508 meters is chosen to test a wide range of conditions. A convergent-divergent nozzle designated as PSU000, shown in Fig. 2, designed at the Pennsylvania State University, is also selected. It has a design Mach number of 1.50 and an exit diameter of 0.0127 meters. This nozzle and associated experimental data are courtesy of Professor D. K. McLaughlin of the Pennsylvania State University. Finally, a dualstream nozzle, shown in Fig. 3, with convergent primary (core) and secondary (fan) streams is selected with primary nozzle exit diameter of 0.0622 meters, secondary nozzle exit inner diameter of 0.101716 meters, and outer diameter of 0.15248 meters. The dualstream nozzle exits are off-set with the secondary stream exit 0.0984 meters upstream of the primary nozzle exit. This Boeing nozzle is selected to help illustrate the effect of the high speed secondary stream on the primary stream's BBSAN sources. This nozzle geometry and associated experimental data are courtesy of Dr. K. Viswanathan of the Boeing Company.

The nozzle operating conditions are summarized in Table 1 for single stream jets and Table 2 for dualstream jets. The case name describes the nozzle used and has an associated run number as a suffix. For each case there are corresponding $N P R$ and $T T R$ values as well as the fully expanded Mach number $M_{j}$. A subscript of $p$ denotes the value for the primary flow or core nozzle and a subscript of $s$ denotes the operating 
condition of the secondary stream or fan flow. These cases are selected based on available experimental data and represent a wide range of over- and under-expanded conditions. In addition, a wide range of $T T R$ are chosen to examine temperature effects on the noise predictions.

SRANS solutions are produced by the Wind-US 2.0 solver for each case shown in Tables 1 and 2. To illustrate the flow-fields produced, a specific result is shown from each nozzle geometry and a single operating condition. In Fig. 4 the SMC000 nozzle is shown with contours of static pressure. The SMC000 nozzle is operating at $M_{j}=1.39$ and $T T R=3.20$. Since the nozzle is operating off-design and under-expanded, Prandtl-Meyer expansion waves form on the nozzle lip. This pressure imbalance leads to the shock cell structure shown. Figure 5 shows the PSU convergent-divergent $M_{d}=1.50$ nozzle operating at $M_{j}=1.30$ and $T T R=2.20$. Contour plots of the streamwise velocity $u$, are shown. In this case the flow is overexpanded and a conical oblique shock wave forms on the nozzle lip and terminates as a barrel (normal) shock. This pressure imbalance also results in a shock cell structure that can be seen in the velocity field. The nature of the flows of over- and under-expanded jets are very different due to the initial oblique shock wave or Prandtl-Meyer expansion wave. Figure 6 shows the Boeing Company nozzle operating at $N P R_{p}=2.40, T T R_{p}=2.70, M_{j p}=1.192, N P R_{s}=1.80, T T R_{s}=1.00$, and $M_{j s}=0.959$. Contours of Mach number are shown. Both the core and fan nozzles are convergent, thus either stream can operate on-design, under-expanded, or subsonically.

It should be noted that the width of a shock wave is typically a few mean free paths and the computational grid can not resolve them. Specific numerical methods have been developed to better capture the shock waves that exist in off-design supersonic jets. Unfortunately, the Wind-US 2.0 solver has no special treatment for shock waves. Standard second order Roe ${ }^{32}$ flux vector splitting is used for spatial discretization and this causes the shocks to become damped prematurely in the downstream direction.

BBSAN must propagate through a very high speed shear layer before it can propagate to the far-field observer. The mean flow has very little effect near the sideline location. In the dualstream case there are essentially two high speed shear layers near the nozzle exits. The source locations of BBSAN can be located by plotting the integrand of the model equations as shown by Miller ${ }^{29}$ or by examining source distribution contour maps from acoustic arrays such as the Deconvolution Approach for the Mapping of Acoustic Sources (DAMAS) created by Brooks et al. ${ }^{33}$ Both of these studies validate the source locations of BBSAN. The effects of these shear layers will be explored in the following jet noise predictions.

Before showing the jet noise predictions the format of their presentation is discussed. The predictions of the two BBSAN models and the two mixing noise models are shown in Figures 7 through 22. Each figure represents jet noise predictions from a nozzle and corresponding operating condition summarized in Table 1 and 2 with corresponding experimental data. The formats of each figure are similar. For example, in Fig. 9 there are six sets of spectra shown and each one represents a different observer position. Observer positions are at one hundred nozzle diameters from the primary nozzle exit at inlet angles, $\Psi$, shown in the upper left hand corner. The downstream direction is represented by values of $\Psi$ greater than ninety degrees. The frequency is non-dimensional in the form of the Strouhal number, $S t$, using the fully expanded jet velocity $u_{j}$ and the fully expanded diameter $D_{j}$. The $y$ axis represents the Sound Pressure Level $(S P L)$ per unit St. This is calculated by adding $10 \log _{10}\left(u_{j} / D_{j}\right)$ to the power spectral density $(S P L$ per unit $\mathrm{Hz}$ ). Each set of experimental data is shown in red, the fine-scale mixing noise predictions of Tam and Auriault ${ }^{24}$ are orange, the fine-scale mixing noise predictions of Morris and Boluriaan ${ }^{25}$ are blue, the BBSAN predictions using Eqn. 54 (see Appendix A) are green (labeled as M5R3), and the newly developed BBSAN model Eqn. 38 are colored black (labeled M6R2). Predictions of the noise produced by the large-scale coherent turbulence are not included in this study. The noise from the large-scale turbulent structures dominate the spectra in the downstream direction. In this direction it is expected that the predictions of BBSAN and fine-scale mixing noise will be much lower than the experimental measurement.

The convergent SMC000 nozzle operating sonically and with $T T R=1.00$ is shown in Fig. 7. Since the jet is not supersonic or off-design there is no BBSAN present. Generally both fine-scale mixing noise predictions over-estimate the fine-scale mixing noise in the sideline and upstream directions. In the downstream direction the large-scale mixing noise dominates the fine-scale mixing noise. In the downstream region, the predictions of fine-scale mixing noise are reduced by approximately four dB relative to the sideline and upstream directions. In Figs. 8 and 9 the same TTR (unheated) is used, and the fully expanded Mach numbers are increased to $M_{j}=1.24$ and $M_{j}=1.39$ respectively. These two cases are operating supersonically, off-design, and unheated. Predictions of the BBSAN using Eqns. 54 and 38 are shown in green and black respectively. At the sideline locations in Figs. 8 and 9 the differences between the two BBSAN models are 
relatively small. This is due to the negligible effects that the shear layer has on the BBSAN propagating in the sideline direction. By examining the differences between the predictions of the two BBSAN models at various angles other than the sideline location, the effects of the mean flow on the BBSAN can be observed. For the cases SMC0002 and SMC0003 in the upstream direction, the half-width of the main BBSAN peak is narrower and generally has a higher rate of decay at lower $S t$ when mean flow effects are included. However, this is a relatively small effect in the single jet case. This can be observed best at angles $\psi=50$ and $\psi=$ 70 degrees. In the downstream direction the opposite is true and the BBSAN decays at a lower rate when mean flow effects are included. At the sideline location the fine-scale mixing effects generally scale well with the experimental data. At the highest $M_{j}$ the fine-scale predictions agree much better compared with the sonic case.

The large-scale turbulent mixing noise in the downstream direction dominates both the BBSAN and fine-scale mixing noise. The predictions do not include the contribution to mixing noise from large-scale coherent turbulence. Since the large-scale turbulent structures are the cause of Mach wave radiation and the large-scale turbulent mixing noise is dominant in almost every case in the downstream direction. For example, in Fig. 7 at $\Psi=150$ degrees the fine-scale mixing noise predictions are dominated by the noise due to large-scale coherent turbulence.

Figure 10 shows the SMC000 nozzle operating at the sonic condition and $T T R=2.70$. This corresponds to the same conditions and predictions shown in Fig. 7 but with heating. The fine-scale mixing predictions of case SMC0004 agree much better with experimental data than SMC0001. This is illustrated at the sideline location where both the models of Tam and Auriault ${ }^{24}$ and Morris and Boluriaan ${ }^{25}$ agree very well at the peak frequency and above. Both models slightly over-predict the noise at low frequencies. In the upstream direction the peak levels are approximately two $\mathrm{dB}$ too high at $\psi=50$. In the downstream direction the large-scale mixing noise dominates the fine-scale mixing noise.

Holding the TTR constant at 2.70, the NPR is increased to obtain fully expanded Mach numbers of 1.24 and 1.39. These experimental results and predictions are shown in Figs. 11 and 12 respectively. Examining the sideline location in Figs. 11 and 12 shows that there is little effect on the BBSAN due to mean flow shear layer effects. At most the predictions differ by one $\mathrm{dB}$ at any $S t$ and have a nearly identical shape. In the upstream direction the mean flow has the effect of narrowing the peaks and there are more significant effects of the mean flow on the BBSAN. At $\psi=110$ degrees the BBSAN model with mean flow effects over-predicts the experimental levels while the absence of mean flow effects causes an under-prediction of the experimental data. This effect is less pronounced for the SMC0006 case relative to the SMC0005 case. Similar effects are also observed in Figs 8 and 9.

The fine-scale turbulent mixing noise in Figs. 11 and 12 follows the same trend seen in Fig. 10. Overprediction by two to four $\mathrm{dB}$ at lower frequencies is shown in the upstream direction in Fig. 12. The peak magnitude of both fine-scale mixing models agrees very well with experimental values in the sideline and upstream direction.

Figures 13 through 15 show the SMC000 nozzle operating at $T T R=3.20$ and $M_{j}=1.00, M_{j}=1.24$, and $M_{j}=1.39$ respectively. Case SMC0007 shown in Fig. 13 is operating sonically and only the fine-scale and large-scale turbulent mixing noise is present in the experimental data. The fine-scale mixing noise models of Tam and Auriault ${ }^{24}$ and Morris and Boluriaan ${ }^{25}$ both perform extremely well. Though over-prediction can be seen in the lower frequencies by up to six dB. The BBSAN in Figs. 13 through 15 demonstrates a similar behavior to the previous predictions. Richer spectral content is evident by the larger number of BBSAN peaks in the upstream direction, as shown in Fig. 15 at $\psi=50$ degrees. In the downstream direction the mean flow has a greater effect on the BBSAN by changing the shape of the spectra and slightly changing its amplitude.

The Penn State convergent-divergent nozzle has a design Mach number of 1.50 and exit diameter of $0.0127 \mathrm{~m}$. Figure 16 shows noise spectra and predictions of the over-expanded jet produced by operating at $M_{j}=1.30$ and $T T R=1.00$. The predictions at $\psi=50$ degrees are not shown because experimental data is not available at this location. This prediction aligns with experimental data at the peak but over-predicts the BBSAN at higher frequencies by about two dB. In the upstream direction the BBSAN peak is narrower, which is similar to the results of the SMC000 convergent nozzle. In the downstream direction, the BBSAN predictions with mean flow effects are highly damped. This is best illustrated at an observer angle of $\psi=150$ degrees. The experimental spectrum at $\psi=130$ degrees shows two peaks of BBSAN. This is not captured with Eqn. 54 that uses the free space Green's function but is captured with Eqn. 38 that uses the adjoint vector Green's function solver. In all cases, the fine-scale mixing noise models under-predict the experiment. 
Recall that shock cells alter the structure of the jet and have a small effect on the fine-scale mixing noise.

Figure 17 shows spectra for an over-expanded jet at $M_{j}=1.30$ and the TTR is increased from 1.00 to 2.20 from the previous case. As in the unheated case, at the sideline location the model of Eqn. 38 is two $\mathrm{dB}$ higher than the unheated case. The spectrum shapes are almost identical. The fine-scale mixing noise under-predicts the experimental spectra at all angles just as in the unheated case. This under-prediction varies by eight to ten $\mathrm{dB}$. The shock cell structure and screech tone can greatly change the two-point fourth order cross-correlation of the turbulent velocity fluctuations. Since the BBSAN predictions use a different set of calibration coefficients for all over-expanded jets relative to under-expanded jets, it is possible that the mixing noise models would show improved accuracy by using a different set of turbulence coefficients for over-expanded jets.

The final two single stream cases are shown in Figs. 18 and 19. The same convergent-divergent PSU nozzle is used but is operating at $M_{j}=1.70$. The spectra in Fig. 18 result from an unheated jet and the spectra in Fig. 19 are for $T T R=2.20$. The addition of heating has eliminated the screech tone and thus altered the BBSAN. Screech tones typically lower the peak BBSAN frequency and raise its amplitude at the sideline location, which is generally free from mean flow propagation effects. This slight effect can be observed by comparing the sideline locations between Figs. 18 and 19. At the sideline locations the shape of the predicted BBSAN spectra are nearly identical but the unheated case is lower by two dB. The fine-scale turbulent mixing noise in the unheated and heated cases under-predict the experiment. In the unheated case the Tam and Auriault ${ }^{24}$ model has better agreement with the experiment than that of Morris and Boluriaan. ${ }^{25}$ In the heated case both models under-predict the fine-scale mixing noise by six to eight $\mathrm{dB}$.

The effect of varying the TTR and holding $M_{j}=1.39$ constant at the sideline location results that at $T T R=1.00$ the peak BBSAN is $115 \mathrm{~dB}$ (Fig. 9), at $T T R=2.70$ it is $118 \mathrm{~dB}$ (Fig. 12), and at TTR $=3.20$ it is $118 \mathrm{~dB}$ (Fig. 15). The saturation of the amplitude of BBSAN with temperature agrees with the recent observations of Viswanathan et al. ${ }^{19}$ and Kuo et al. ${ }^{18}$ The relative independence of BBSAN scaling with respect to stagnation temperature was first observed by Harper-Bourne and Fisher. ${ }^{2}$ In all three cases, the BBSAN models are under-predicting the peak level and over-predicting the peak frequency. Screech is present at all TTR and will raise the BBSAN levels by up to three to four $\mathrm{dB}$ while lowering the peak frequency relative to the non-screeching jet. The temperature correction factor of $\operatorname{Tam}^{10}$ has been used to account for the saturation of BBSAN with increasing temperature for Eqns. 38 and 54.

The effect of varying the $T T R$ and holding $M_{j}=1.00$ at the sideline location shows that at $T T R=1.00$ the peak mixing noise is $88 \mathrm{~dB}$, at $T T R=2.70$ it is $100 \mathrm{~dB}$, and at $T T R=3.20$ it is $103 \mathrm{~dB}$. The predictions of Tam and Auriault ${ }^{24}$ and Morris and Boluriaan ${ }^{25}$ agree very well at the sideline locations in terms of absolute magnitude for the heated cases. Both of these models have shown excellent scaling with experimental data. The slight over-prediction of Morris and Boluriaan ${ }^{25}$ and the over-prediction by Tam and Auriault ${ }^{24}$ by six decibels for the unheated case, causes some concern about the scaling of turbulent kinetic energy and dissipation in the Menter ${ }^{28}$ SST turbulent model.

The predictions and experimental spectra of dualstream jets are now examined. Both the core and fan nozzles are convergent as shown in Fig. 3. Figure 20 shows the jet noise spectra of the dual stream nozzle operating at $M_{j p}=0.710, M_{j s}=1.358, T T R_{p}=2.14$, and $T T R_{s}=1.00$. These operating conditions give a heated subsonic core-flow and supersonic off-design fan-flow. The shock cell structure of the fan-flow is contained by the coannular fan stream between the ambient environment and the core-flow. Thus, there are two regions of BBSAN sources. It has been shown by Miller and Morris ${ }^{16}$ that the dominant BBSAN source location in this type of dualstream jet is the fan-stream ambient shear layer. However, this does not imply that there are no BBSAN contributions from the core-flow shear layer. The mean flow effects on the BBSAN sources contained in the fan-flow shear layer are identical to those of singlestream jets. The mean flow effects on the core flow shear layer are much greater because it is surrounded by the high-speed secondary flow.

Examining the sideline location of Fig. 20, the predictions of the BBSAN can be seen with and without mean flow effects. The prediction with mean flow effects, Eqn. 38, has an improved spectrum shape near the main BBSAN peak compared to the prediction of Eqn. 54. Note that the jet is screeching and this affects the spectral shape of BBSAN, making the experimental data have narrower peaks than its non-screeching counterpart. Inclusion of the mean flow effects causes a more rapid decay of BBSAN at low frequencies and a more gradual fall-off at higher frequencies relative to predictions of Eqn. 54. This trend can be seen at the other observer angles. In the upstream direction, the effect of including the mean flow has caused the BBSAN prediction to better capture the existence of a second BBSAN peak.

Fine-scale mixing noise predictions are also shown in Fig. 20. At all observer angles the prediction of 
Tam and Auriault ${ }^{24}$ is higher than Morris and Boluriaan ${ }^{25}$ but has very similar spectral shapes. At the sideline location the model of Tam and Auriault ${ }^{24}$ over-predicts the mixing noise by approximately fifteen $\mathrm{dB}$ and the prediction of Morris and Boluriaan ${ }^{25}$ over-predicts by seven $\mathrm{dB}$.

Figure 21 shows predictions for the Boeing coannular nozzle operating at $M_{j p}=1.281, M_{j s}=0.848$, $T T R_{p}=2.870$, and $T T R_{s}=1.00$. The core flow is hotter than the previous case and is now operating supersonic and off-design. The secondary fan plume is high speed subsonic flow and contains no BBSAN sources. The BBSAN spectra are very similar across all observer positions except at high inlet angles. The lower frequency BBSAN is very flat in the upstream direction. The amplitudes of BBSAN predictions using Eqn. 38 at the sideline and upstream directions show better agreement with the experiment compared to BBSAN predictions using Eqn. 54. Unlike the previous dualstream case, the fine-scale mixing noise predictions are lower than the experimental data, but predict the peak frequencies correctly. The model of Tam and Auriault ${ }^{24}$ under-predicts the noise at the sideline location by two $\mathrm{dB}$ and the prediction of Morris and Boluriaan ${ }^{25}$ under-predicts the noise at the sideline location by eight $\mathrm{dB}$.

A final case is examined where both the core and fan streams are supersonic. The Boeing dualstream nozzle operates at $M_{j p}=1.281, M_{j s}=1.358, T T R_{p}=2.870$, and $T T R_{s}=1.00$ and the noise spectra are shown in Fig. 22. This case (DUAL7) is selected because it combines the difficulties inherent in the two previous dualstream cases. At the sideline location the predictions of Eqn. 38 and Eqn. 54 show slightly better agreement relative to magnitude and a much richer spectral content. Multiple BBSAN peaks can be seen at the higher BBSAN harmonics. In the downstream direction similar results are seen compared to previous BBSAN predictions relative to experimental data. The data in the upstream direction, particularly at $\psi=50$ degrees, represents the most important result of this paper. The BBSAN predictions of Eqn. 38 shows remarkable agreement with experimental data compared to the prediction of Eqn. 54. The predicted spectrum that includes the vector Green's function has much richer spectral content, the correct fall-off at low and high frequencies, and excellent amplitude agreement until $S t=11$. The mixing noise predictions are similar to those of Fig. 20.

\section{Discussion and Conclusion}

An acoustic analogy based on the Euler equations has been formed for BBSAN. The parameter values for the model are provided by a steady mean flow solution of the RANS equations and the vector Green's function of the linearized Euler equations using an adjoint approach. This acoustic analogy reduces to the model developed by Morris and Miller ${ }^{1}$ if the vector Green's function of the linearized Euler equations for a quiescent environment is used. Comparison of these two models shows that at the sideline location the spectral shape is the same. Thus, the mean flow effects are minimal if the observer is at the sideline location of the jet. In the downstream region the mean flow effects on BBSAN generally decrease the noise. In the upstream direction the mean flow effects have more significance and the newly developed BBSAN model shows better agreement with experimental data than predictions that neglect mean flow effects. The newly developed model predictions show an increased number of broadband peaks, slightly better agreement in amplitude relative to experimental results, faster decay at lower frequencies and slower decay at higher frequencies relative to the peak frequency. These mean flow effects, although slight, generally yield more accurate predictions relative to model of Morris and Miller. ${ }^{1}$ In particular, the BBSAN calculations for the dualstream jets in the upstream direction show the most improvement.

It is important to note that one of the assumptions in the formulation of the adjoint vector Green's function solver is that the static pressure everywhere in the jet is equal to the ambient. Among a few other assumptions, this assumption is necessary in order to form a single second order differential equation for the pressure from the Euler equations. This assumption is valid for all subsonic jets and on-design supersonic jets. Off-design supersonic jets have a shock cell structure due to the static pressure at the nozzle exit not matching the ambient static pressure. The resultant shocks and Prandtl-Meyer expansions cause the static pressure to be either much greater or much smaller than the ambient values. Thankfully, the BBSAN sources exist near the outside of the jet in the shear layer and the pressure mismatch at these locations is very small. The sources of fine-scale mixing noise exist in the high speed shear layer, the transition region, and the fully developed region of the jet and are much more effected by this assumption. It is perhaps for this reason that some of the mixing noise calculations of Tam and Auriault ${ }^{24}$ and Morris and Boluriaan ${ }^{25}$ differ so much in amplitude relative to the experimental data. If a numerical adjoint vector Green's function solver of the linearized Euler equations is available that makes no assumption concerning the mean flow (in particular the 
static pressure of the jet being equal to the ambient) and make use of the SRANS calculations, then it is expected that the fine-scale mixing noise predictions and BBSAN predictions of Eqn. 38 would have better agreement with experiment.

The predictions do not include the contribution to mixing noise from large-scale coherent turbulence. Since the large-scale turbulent structures are the cause of Mach wave radiation and the large-scale turbulent mixing noise is dominant in almost every case in the downstream direction, a model needs to be constructed for this purpose. The large-scale coherent turbulent structures are a time dependent phenomenon that is highly dependent on the disturbances in the jet shear layer and the stability of the shear layer near the nozzle exit. Also, these large-scale coherent turbulent structures are modeled in the development of the BBSAN equations by Gaussian two-point space-time correlations. Thus, a Large Eddy Simulation (LES) which captures the large-scale structures and filters out the small scale structures may yield accurate predictions of the Mach wave radiation without the need to capture the noise from fine-scale turbulence, for example, see Shur et al. ${ }^{34}$

The modular nature of RISN and the locally parallel adjoint vector Green's solver for the linearized Euler equations allowed implementation of the fine-scale mixing noise models of Tam and Auriault ${ }^{24}$ and Morris and Boluriaan ${ }^{25}$ with ease. In general the fine-scale turbulent mixing noise models capture the peak frequencies, peak amplitudes, and fall-off at high frequencies. At lower frequencies the models generally had too slow a spectral fall-off relative to experimental data. This could easily be corrected with changes described in the appendix of Morris and Boluriaan. ${ }^{25}$ In the downstream direction the fine-scale mixing noise is dominated by the large-scale mixing noise. Thus, the fine-scale mixing noise models have little contribution to the total noise in this direction.

The developed acoustic analogies for BBSAN and fine-scale mixing noise make use of a vector Green's function. The arguments of these models could easily be found by a numerical adjoint vector Green's function solver that makes no assumption about the flow-field or the geometry. In fact, use of a tailored vector Green's function could include the effects of reflection and refraction of the airframe, refraction due to the jet shear layer, and propagation to the far-field without further modification to the acoustic analogies.

\section{Appendix A}

It will now be shown that Eqn. 38 reduces to the model equation of Morris and Miller. ${ }^{1}$ The mean flow effects are neglected by using the Green's function of the Helmholtz equation. This can be performed by setting Eqns. 10 and 11 mean flow conditions to the ambient values, taking the Fourier transform with respect to time, and finding the Green's function. This yields,

$$
g(\underline{x}, \underline{z}, \omega)=\frac{-1}{4 \pi c_{\infty}^{2}|\underline{x}-\underline{z}|} \exp \left[i \omega|\underline{x}-\underline{z}| / c_{\infty}\right]
$$

where $\underline{z}$ is a vector to the source locations. The vector Green's function of the linearized Euler equations for a quiescent environment based on Eqns. 10 and 11 are,

$$
\pi_{g}^{n}(\underline{x}, \underline{y}, \omega)=\delta_{i n} \int_{\underline{z}} \frac{\partial}{\partial z_{i}} \delta(\underline{z}-\underline{y}) g(\underline{x}, \underline{z}, \omega) d \underline{z}
$$

Substituting Eqn. 50 into Eqn. 51 and simplifying the previous equation yields an expression for the vector Green's function in the absence of varying mean flow (which is very similar to the Green's function of the Helmholtz equation),

$$
\pi_{g}^{n}(\underline{x}, \underline{y}, \omega)=-\frac{i \omega x_{n}}{4 \pi c_{\infty}^{3} x^{2}} \exp \left[i \omega x / c_{\infty}\right]
$$

Substituting Eqn. 52 into Eqn. 38 yields,

$$
\begin{aligned}
S(\underline{\mathrm{x}}, \omega)=\frac{1}{16 \pi^{3 / 2} c_{\infty}^{4} x^{2}} \int_{-\infty}^{\infty} \ldots \int_{-\infty}^{\infty} & \sum_{n=1}^{3} \sum_{m=1}^{3} a_{n m} \frac{x_{n} x_{m}}{x^{2}} \omega^{2} \exp \left[i k_{1} y_{1}\right] \frac{K l_{\perp}^{2} \tau_{s}}{l} p_{s}(\underline{\mathrm{y}}) \tilde{p}_{s}\left(k_{1}, y_{2}, y_{3}\right) \\
& \times \frac{\exp \left[-l^{2}\left(k_{1}-\omega \cos \theta / c_{\infty}\right)^{2} / 4-\omega^{2} l_{\perp}^{2} \sin ^{2} \theta /\left(4 c_{\infty}^{2}\right)\right]}{1+\left(1-M_{c} \cos \theta-u k_{1} / \omega\right)^{2} \omega^{2} \tau_{s}^{2}} d k_{1} d \underline{y}
\end{aligned}
$$


This expression for the spectral density provides no account for the mean flow effects but retains the ability to specify anisotropic turbulence. Morris and Miller ${ }^{1}$ used the Proudman ${ }^{35}$ form for isotropic turbulence, $a_{n m} x_{n} x_{m} / x^{2}=1$. Using this assumption and simplifying yields,

$$
\begin{array}{r}
S(\underline{\mathrm{x}}, \omega)=\frac{1}{16 \pi^{3 / 2} c_{\infty}^{4} x^{2}} \int_{-\infty}^{\infty} \ldots \int_{-\infty}^{\infty} \frac{K l_{\perp}^{2}}{l \tau_{s}} p_{s}(\underline{\mathrm{y}}) \tilde{p}_{s}\left(k_{1}, y_{2}, y_{3}\right) \exp \left[i k_{1} y_{1}\right] \\
\times \tau_{s}^{2} \omega^{2} \frac{\exp \left[-l^{2}\left(k_{1}-\omega \cos \theta / c_{\infty}\right)^{2} / 4-\omega^{2} l_{\perp}^{2} \sin ^{2} \theta /\left(4 c_{\infty}^{2}\right)\right]}{1+\left(1-M_{c} \cos \theta-u k_{1} / \omega\right)^{2} \tau_{s}^{2} \omega^{2}} d k_{1} d \underline{y}
\end{array}
$$

which is the model equation developed in Morris and Miller. ${ }^{1}$ This equation is applicable to three-dimensional flow-fields, but has the simplifications of isotropic turbulence and no effects of BBSAN propagation induced by variations of the shear layer mean flow.

\section{Acknowledgements}

This research represents an informal collaboration between The National Aeronautics and Space Administration (NASA) Langley Research Center (LaRC) and The Pennsylvania State University. The first author is grateful for continuous support from the NASA Fundamental Aeronautics Program (FAP) Supersonics Project. The authors are grateful for the experimental data provided by Dr. K. Viswanathan of the Boeing Company, Professor Dennis K. McLaughlin of the Pennsylvania State University, and the jet noise group of NASA Glenn Research Center at Lewis Field.

\section{References}

\footnotetext{
${ }^{1}$ Morris, P. J. and Miller, S. A. E., "Prediction of Broadband Shock-Associated Noise Using Reynolds-Averaged NavierStokes Computational Fluid Dynamics," AIAA Journal, Vol. 48, No. 12, 2010, pp. 2931-2961.

${ }^{2}$ Harper-Bourne, M. and Fisher, M. J., "The noise from Shock-Waves in Supersonic Jets," AGARD, 1973.

${ }^{3}$ SAE International, SAE ARP876, Revision D. Gas Turbine Jet Exhaust Noise Prediction, SAE International, Warrendale, PA, 1994.

${ }^{4}$ Zorumski, W. E., "Aircraft noise prediction program. Theoretical manual. Parts 1 and 2," NASA TM 83199, 1982.

${ }^{5}$ Tam, C. K. W., "Stochastic model Theory of Broadband Shock-Associated Noise from Supersonic Jets," Journal of Sound and Vibration, 1987, pp. 265-302.

${ }^{6}$ Tam, C. K. W. and Tanna, H. K., "Shock-Associated Noise of Supersonic Jets from Convergent-Divergent Nozzles," J. Sound Vib., Vol. 81, No. 3, 1982, pp. 337-358.

${ }^{7}$ Pack, D. C., "A note on Prandtls Formula for the Wavelength of a Supersonic Gas Jet," Quarterly Journal of Applied Mathematics and Mechanics, Vol. 3, 1950, pp. 173-181.

${ }^{8}$ Tam, C. K. W., Jackson, J. A., and Seiner, J. M., "A Multiple-Scales Model of the Shock-Cell Structure of Imperfectly Expanded Supersonic Jets," Journal of Fluid Mechanics, Vol. 153, 1985, pp. 123-149.

${ }^{9}$ Tam, C. K. W. and Chen, K. C., "A Statistical Model of Turbulence in Two-Dimensional Mixing Layers," Journal of Fluid Mechanics, Vol. 92, 1979, pp. 303-326.

${ }^{10}$ Tam, C. K. W., "Broadband Shock-Associated Noise of Moderately Imperfectly-Expanded Supersonic Jets," Journal of Sound and Vibration, Vol. 140, No. 1, 1990, pp. 55-71.

${ }^{11}$ Morris, P. J. and Miller, S. A. E., "The Prediction of Broadband Shock-Associated Noise Using RANS CFD," AIAA Paper 2009-3315, 2009.

${ }^{12}$ Ribner, H. S., "The Generation of Sound by Turbulent Jets, Advances in Applied Mechanics," International Journal of Aeroacoustics, Vol. 8, 1964, pp. 104-182.

${ }^{13}$ Abdelhamid, Y. A. and Ganz, U. W., "Prediction of Shock-Cell Structure and Noise in Dual Flow Nozzles," AIAA Paper 2007-3721, 2007.

${ }^{14}$ Tam, C. K. W., Pastouchenko, N., and Viswanathan, K., "Broadband Shock-Cell Noise from Dual Stream Jets," AIAA Paper 2008-2833, 2008.

${ }^{15}$ Tam, C. K. W. and Reddy, N. N., "Prediction Method for Broadband Shock-Associated Noise from Supersonic Rectangular Jets," Journal of Aircraft, Vol. 33, 1996, pp. 298-303.

${ }^{16}$ Miller, S. A. E. and Morris, P. J., "The Prediction of Broadband Shock-Associated Noise from Dualstream and Rectangular Jets Using RANS CFD," 16th AIAA/CEAS Aeroacoustics Conference AIAA 2010-3730, 2010.

${ }^{17}$ Deneuville, P., "Simplied Prediction Method of Shock Cell Noise of Supersonic Jets from Conical Nozzles," SNECMA YKA No. 5982/76, Societe Nationale dEdute et de Construction de Moteurs dAvion, 1976.

${ }^{18}$ Kuo, C., McLaughlin, D. K., and Morris, P. J., "Effects of Supersonic Jet Conditions on Broadband Shock-Associated Noise," 49th AIAA Aerospace Sciences Meeting including the New Horizons Forum and Aerospace Exposition AIAA 2011-1032, 2011.

${ }^{19}$ Viswanathan, K., Alkislar, M. B., and Czech, M. J., "Characteristics of the Shock Noise Component of Jet Noise," AIAA Journal, Vol. 48, No. 1, 2010, pp. 25-46.
} 
${ }^{20}$ Dahl, M. D., "Predictions of Supersonic Jet Mixing and Shock-Associated Noise Compared with Measured Far-Field Data," NASA TM-2010-216328, 2010.

${ }^{21}$ Tam, C. K. W. and Auriault, L., "Mean flow refraction effects on sound radiated from localized sources in a jet," Journal of Fluid Mechanics, Vol. 370, 1998, pp. 149-174.

${ }^{22}$ Raizada, N., "Numerical Prediction of Noise from High Speed Subsonic Jets using an Acoustic Analogy," Ph.D. Dissertation The Pennsylvania State University, December 2005.

${ }^{23}$ Khavaran, A., Bridges, J., and Georgiadis, N., "Prediction of Turbulence-Generated Noise in Unheated Jets," NASA TM-2005-213827, 2005.

${ }^{24}$ Tam, C. K. W. and Auriault, L., "Jet Mixing Noise from Fine-Scale Turbulence," AIAA Journal, Vol. 37, No. 2, 1999, pp. $145-153$.

${ }^{25}$ Morris, P. J. and Boluriaan, S., "The Prediction of Jet Noise From CFD Data," 10th AIAA/CEAS Aeroacoustics Conference, 2004-2977.

${ }^{26}$ Karabasov, S. A., Hynes, T. P., and Dowling, A. P., "Effect of Mean-Flow Evolution on Sound Propagation through Non-Uniform Jet Flows," 13th AIAA/CEAS Aeroacoustics Conference AIAA-2007-3655, 2007.

${ }^{27}$ Karabasov, S. A., Afsar, M. Z., Hynes, T. P., Dowling, A. P., McMullan, W. A., Pokora, C. D., Page, G. J., and McGuirk, J. J., "Jet Noise: Acoustic Analogy Informed by Large Eddy Simulation," AIAA Journal, Vol. 48, No. 7, 2010 , pp. 1312-1325.

${ }^{28}$ Menter, F. R., "Two-Equation Eddy-Viscosity Turbulence Models for Engineering Applications," AIAA Journal, Vol. 32, No. 8, 1994, pp. $1598-1605$.

${ }^{29}$ Miller, S. A. E. and Veltin, J., "Assessment of Computational Fluid Dynamics for Supersonic Shock Containing Jets," AIAA Journal, Vol. 47, No. 11, 2009, pp. $2738-2746$.

${ }^{30}$ Tam, C. K. W. and Ganesan, A., "Modified k - epsilon Turbulence Model for Calculating Hot Jet Mean Flows and Noise," AIAA Journal, Vol. 41, No. 1, 2004, pp. 26-34.

${ }^{31}$ Miller, S. A. E. and Veltin, J., "Experimental and Numerical Investigation of Flow Properties of Supersonic Helium-Air Jets," AIAA Journal, Vol. 49, No. 1, 2011, pp. 235-246.

${ }^{32}$ Roe, P. L., "Approximate Riemann Solvers, Parameter Vectors, and Difference Schemes," Journal of Computational Physics, Vol. 135, No. 2, 1997, pp. 357-372.

${ }^{33}$ Brooks, T., Humphreys, W., and Plassman, G., "DAMAS Processing for a Phased Array Study in the NASA Langley Jet Noise Laboratory," AIAA-2010-3780, 2010.

${ }^{34}$ Shur, M. L., Spalart, P. R., and Strelets, M. K., "LES-Based Noise Prediction for Shocked Jets in Static and Flight Conditions," AIAA/CEAS Aeroacoustics Conference AIAA 2010-3840, 2010.

${ }^{35}$ Proudman, J., "The Generation of Noise by Isotropic Turbulence," Proc. Roy. Soc. A., Vol. 214 , No. $119,1952$. 


\section{Tables}

Table 1. Nozzle operating conditions for single stream jets.

\begin{tabular}{c|rrr} 
Case & $N P R$ & $M_{j}$ & $T T R$ \\
\hline SMC0001 & 1.893 & 1.00 & 1.00 \\
SMC0002 & 2.556 & 1.24 & 1.00 \\
SMC0003 & 3.154 & 1.39 & 1.00 \\
SMC0004 & 1.893 & 1.00 & 2.70 \\
SMC0005 & 2.556 & 1.24 & 2.70 \\
SMC0006 & 3.154 & 1.39 & 2.70 \\
SMC0007 & 1.893 & 1.00 & 3.20 \\
SMC0008 & 2.556 & 1.24 & 3.20 \\
SMC0009 & 3.154 & 1.39 & 3.20 \\
PSU0001 & 2.770 & 1.30 & 1.00 \\
PSU0002 & 4.930 & 1.70 & 1.00 \\
PSU0003 & 2.770 & 1.30 & 2.20 \\
PSU0004 & 4.930 & 1.70 & 2.20
\end{tabular}

Table 2. Nozzle operating conditions for dual stream jets.

\begin{tabular}{c|rrrrrr} 
Case & $N P R_{p}$ & $M_{j p}$ & $T T R_{p}$ & $N P R_{s}$ & $M_{j s}$ & $T T R_{s}$ \\
\hline DUAL4 & 1.400 & 0.710 & 2.14 & 2.140 & 1.358 & 1.00 \\
DUAL5 & 2.700 & 1.281 & 2.87 & 2.870 & 0.848 & 1.00 \\
DUAL7 & 2.556 & 1.281 & 2.87 & 2.870 & 1.358 & 1.00
\end{tabular}




\section{Figures}

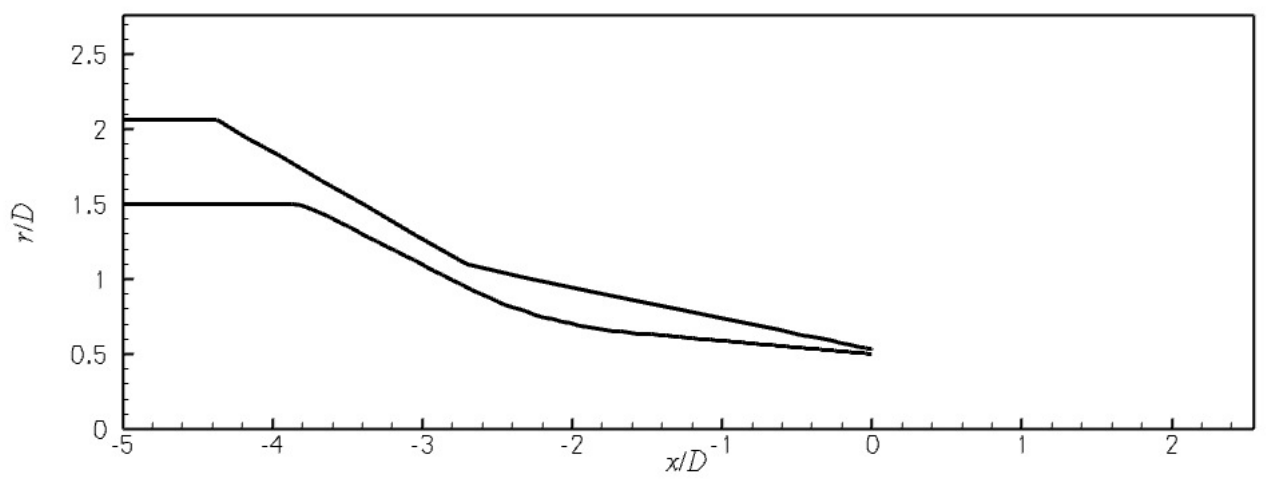

Figure 1. The nozzle contour of the singlestream convergent SMC000 nozzle. $D=0.0508 \mathrm{~m}$.

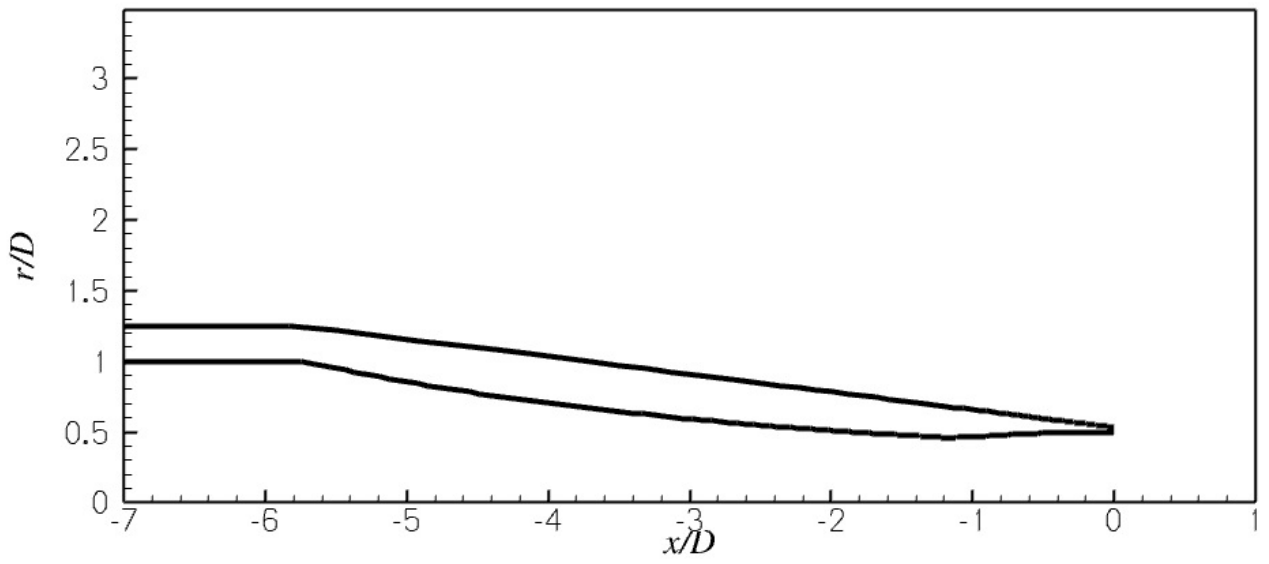

Figure 2. The nozzle contour of the singlestream convergent-divergent PSU nozzle. $D=0.0127 \mathbf{m}$. 


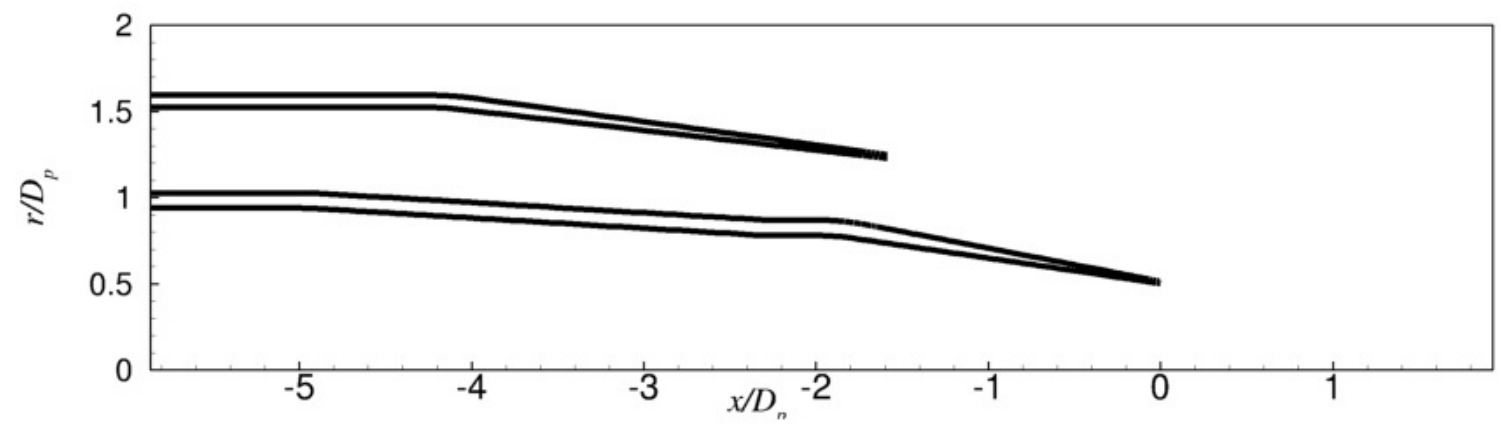

Figure 3. The nozzle contour of the dual stream nozzle. The core nozzle exit diameter is, $D_{p}=0.0622 \mathrm{~m}$, the fan nozzle exit inner diameter is $0.101716 \mathbf{~ m}$, and fan nozzle exit outer diameter, $D_{s}=0.15248 \mathrm{~m}$.

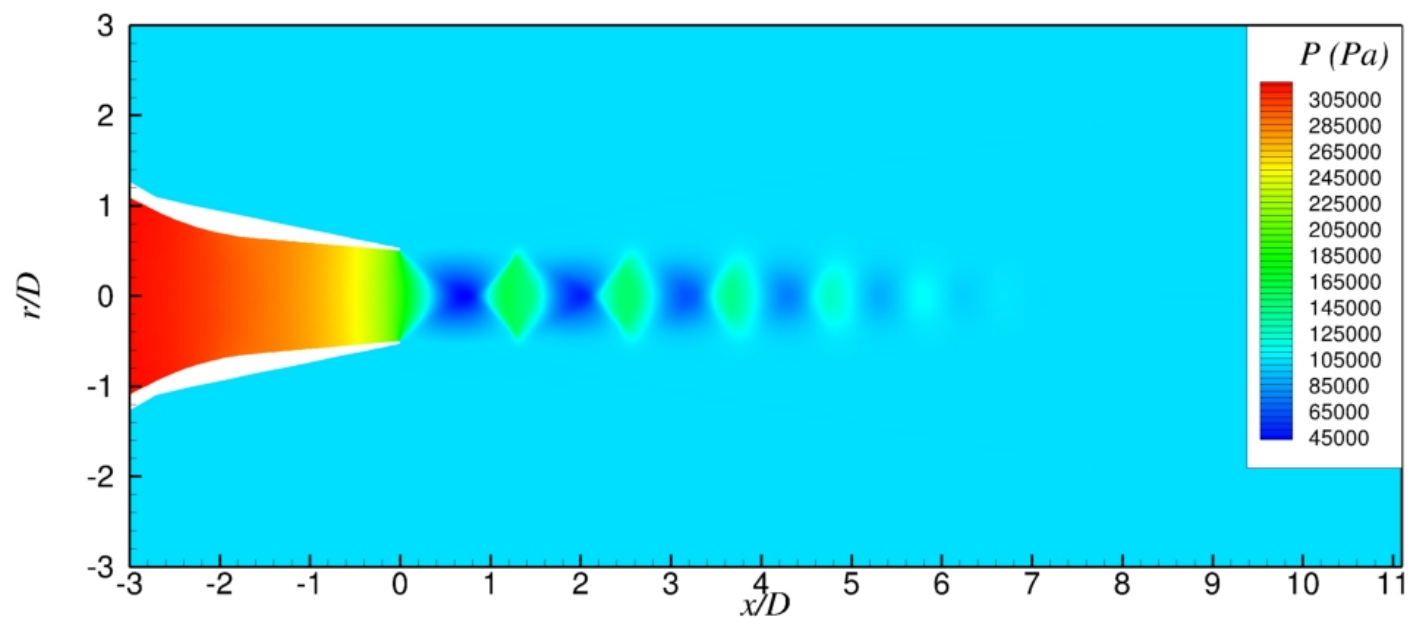

Figure 4. Static pressure contour plot of the singlestream convergent SMC nozzle. The primary flow is under-expanded and supersonic operating at $M_{j}=1.39$ and $T T R=3.20$.

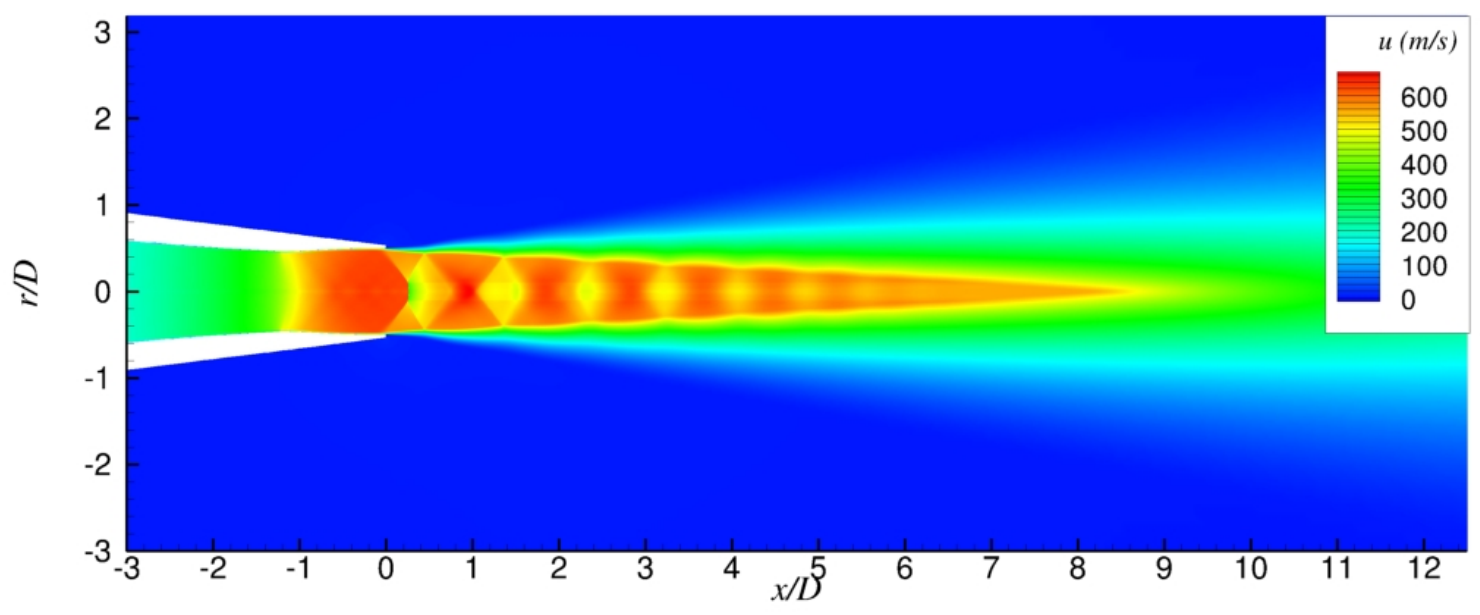

Figure 5. Streamwise velocity, $u$, contour plot of the convergent-divergent PSU nozzle. The jet flow is overexpanded and supersonic operating at $M_{j}=1.30$ and $T T R=2.20$. 


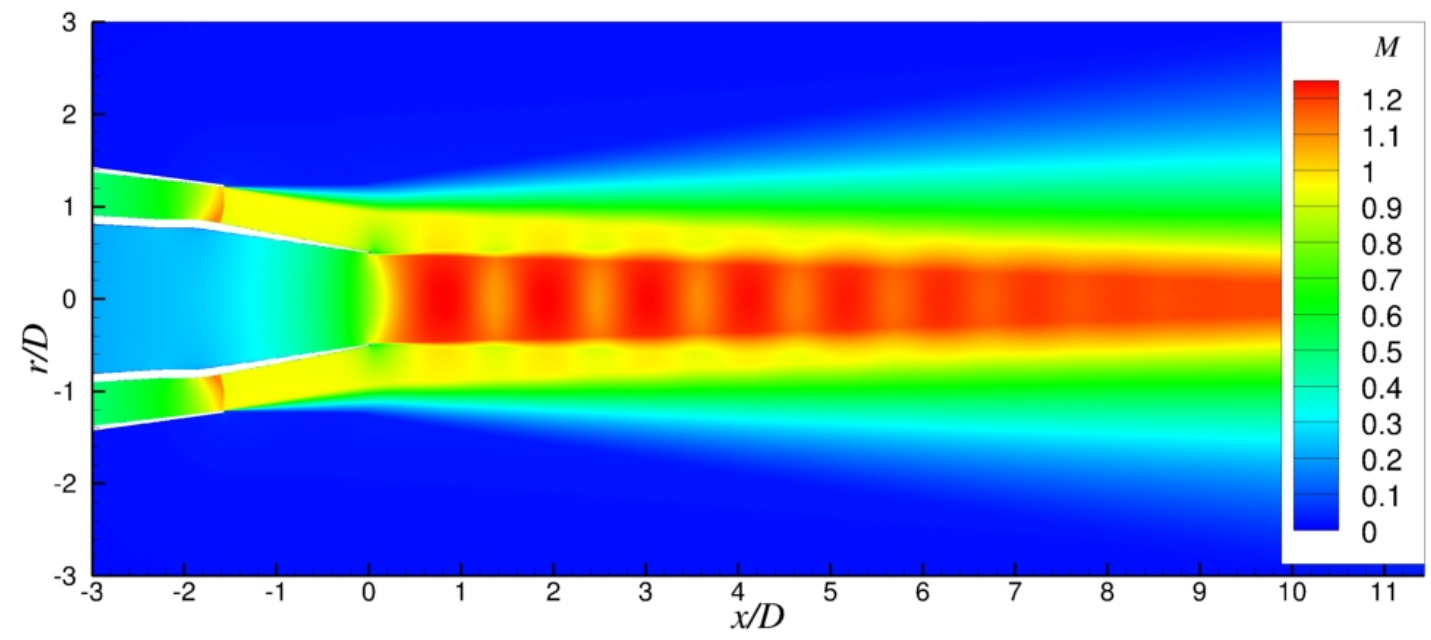

Figure 6. Mach number contour plot of the dualstream nozzle. The primary flow is under-expanded and supersonic operating at $M_{j}=1.192$ and $T T R=2.70$ and the secondary flow is operating at $M_{j}=0.959$ and $T T R=1.00$. 

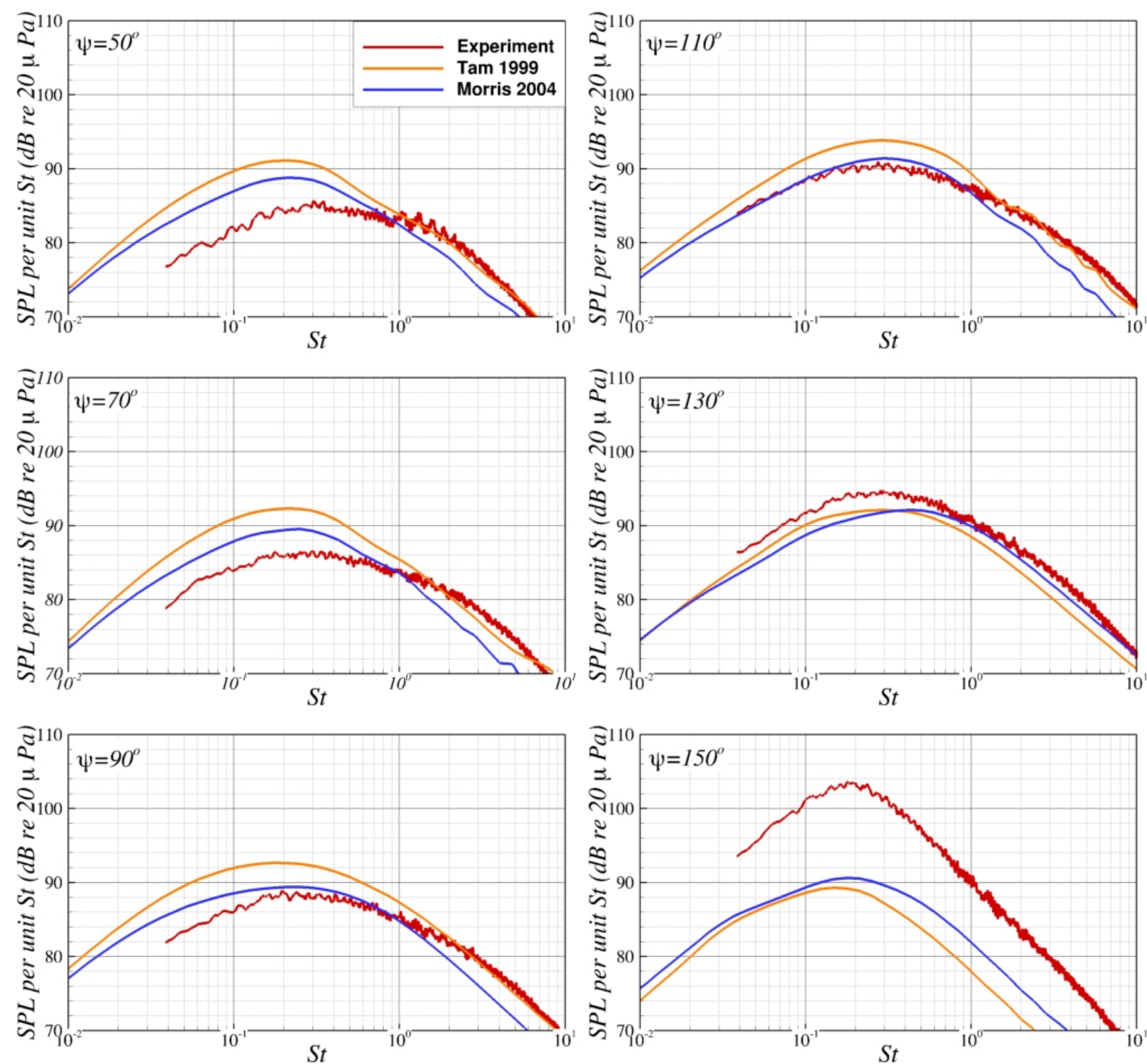

Figure 7. The noise spectra from the SMC000 nozzle case SMC0001, $M_{d}=1.00 D=0.0508$ m, with operating conditions $M_{j}=1.00$ and $T T R=1.00$, at a distance of $R / D=100$ and observer angles $\Psi$. 

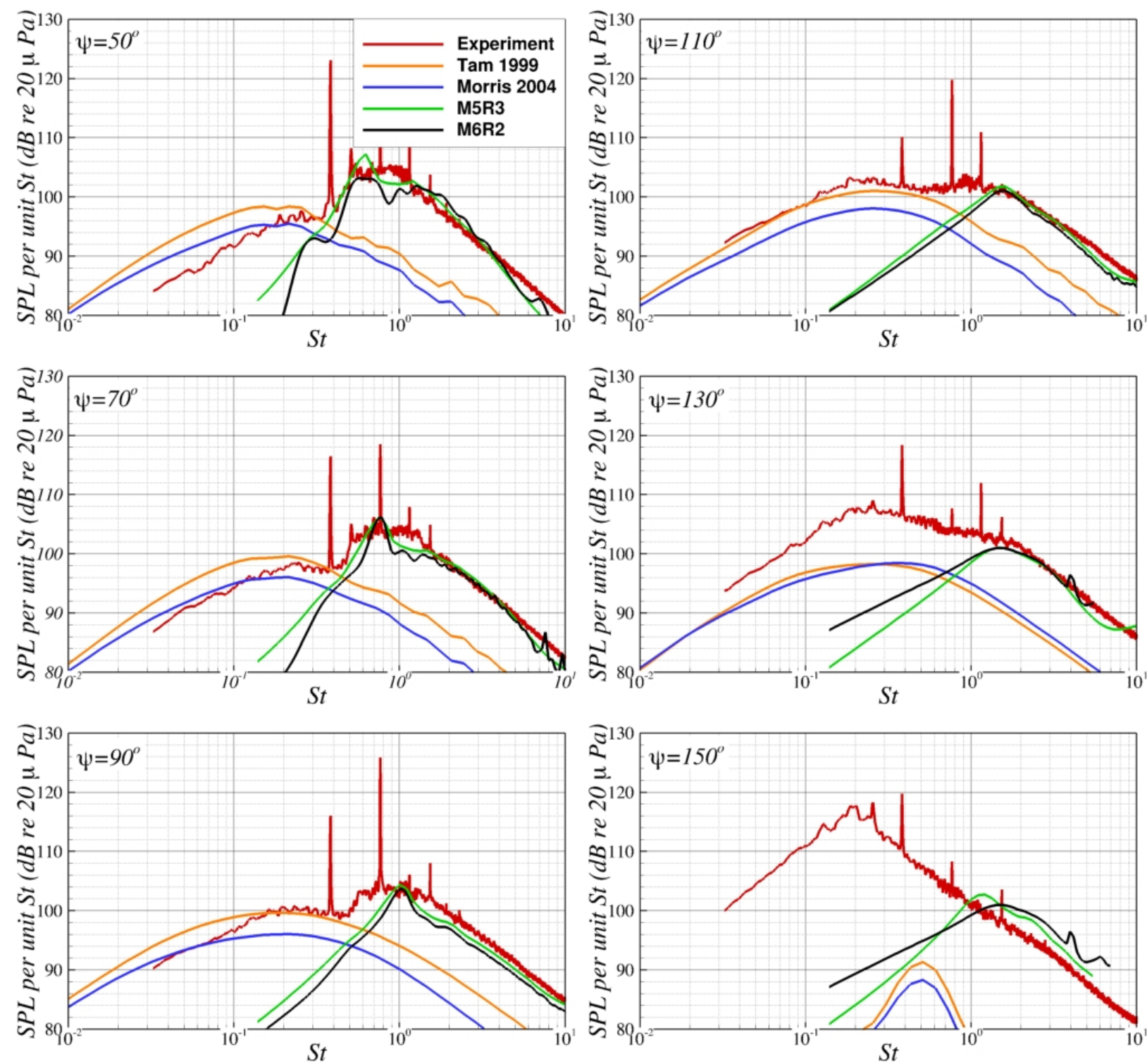

Figure 8. The noise spectra from the SMC000 nozzle case SMC0002, $M_{d}=1.00 D=0.0508$ m, with operating conditions $M_{j}=1.24$ and $T T R=1.00$, at a distance of $R / D=100$ and observer angles $\Psi$. 

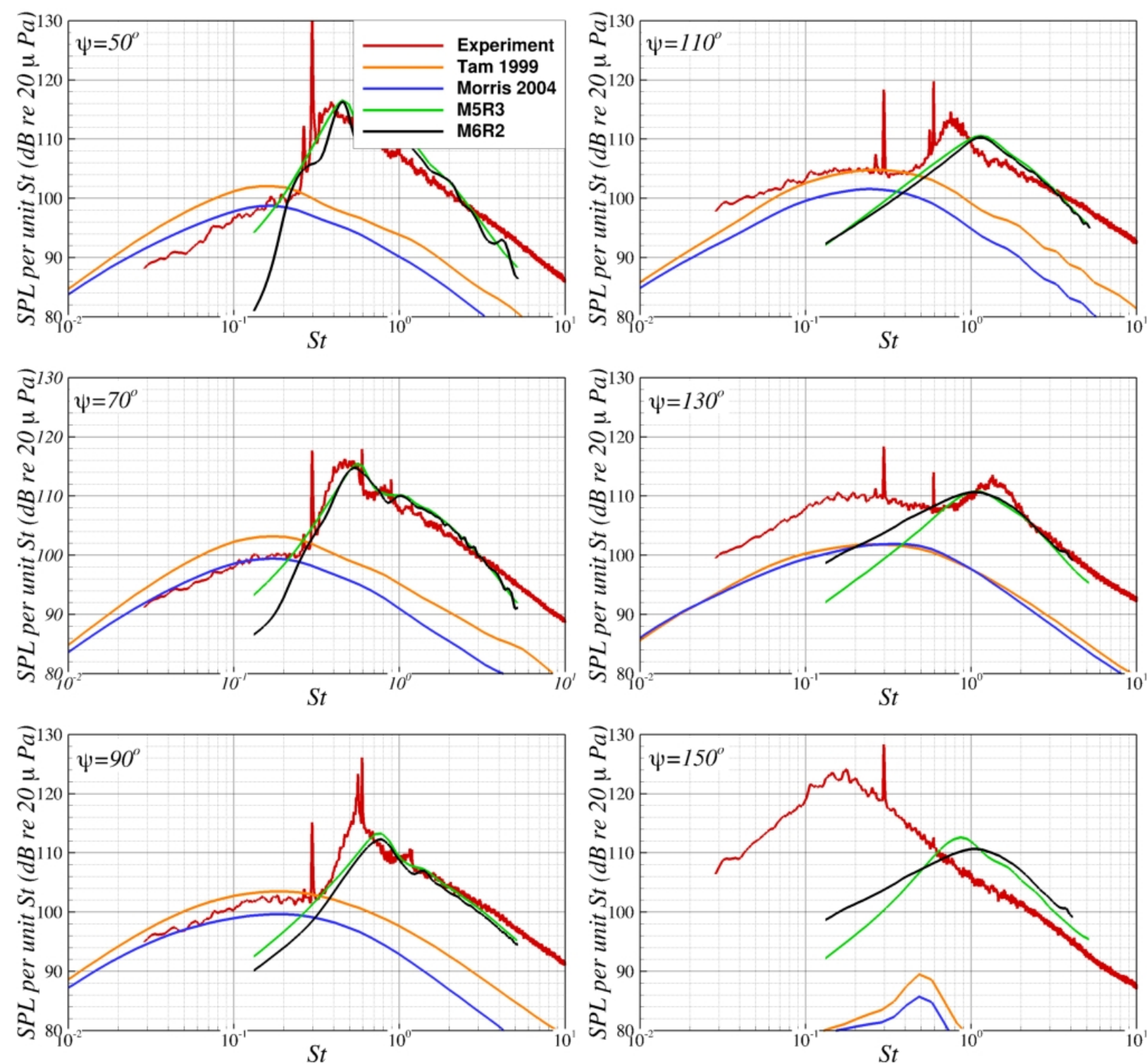

Figure 9. The noise spectra from the SMC000 nozzle case SMC0003, $M_{d}=1.00 D=0.0508$ m, with operating conditions $M_{j}=1.39$ and $T T R=1.00$, at a distance of $R / D=100$ and observer angles $\Psi$. 

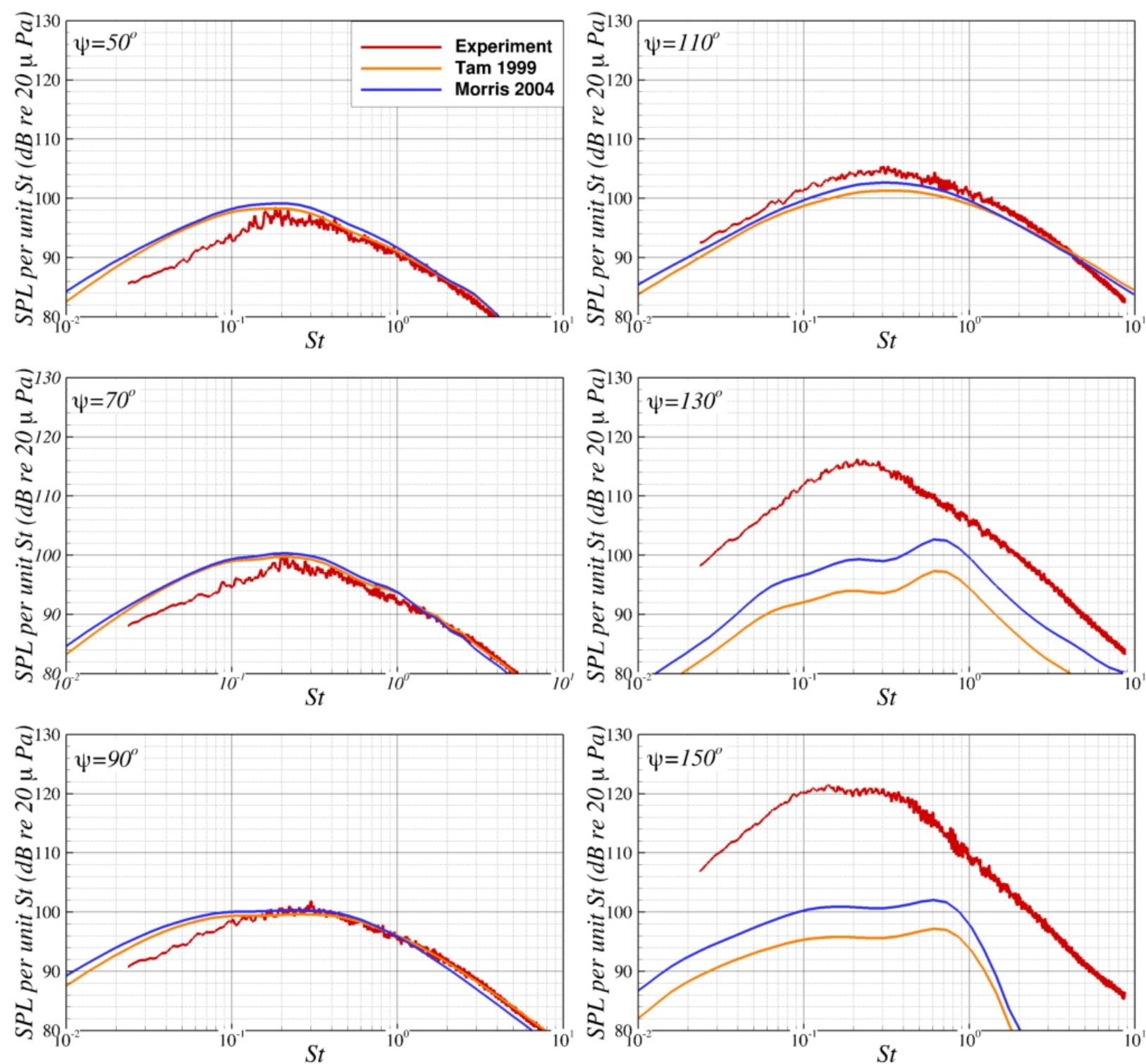

Figure 10. The noise spectra from the SMC000 nozzle case SMC0004, $M_{d}=1.00 D=0.0508 \mathrm{~m}$, with operating conditions $M_{j}=1.00$ and $T T R=2.70$, at a distance of $R / D=100$ and observer angles $\Psi$. 

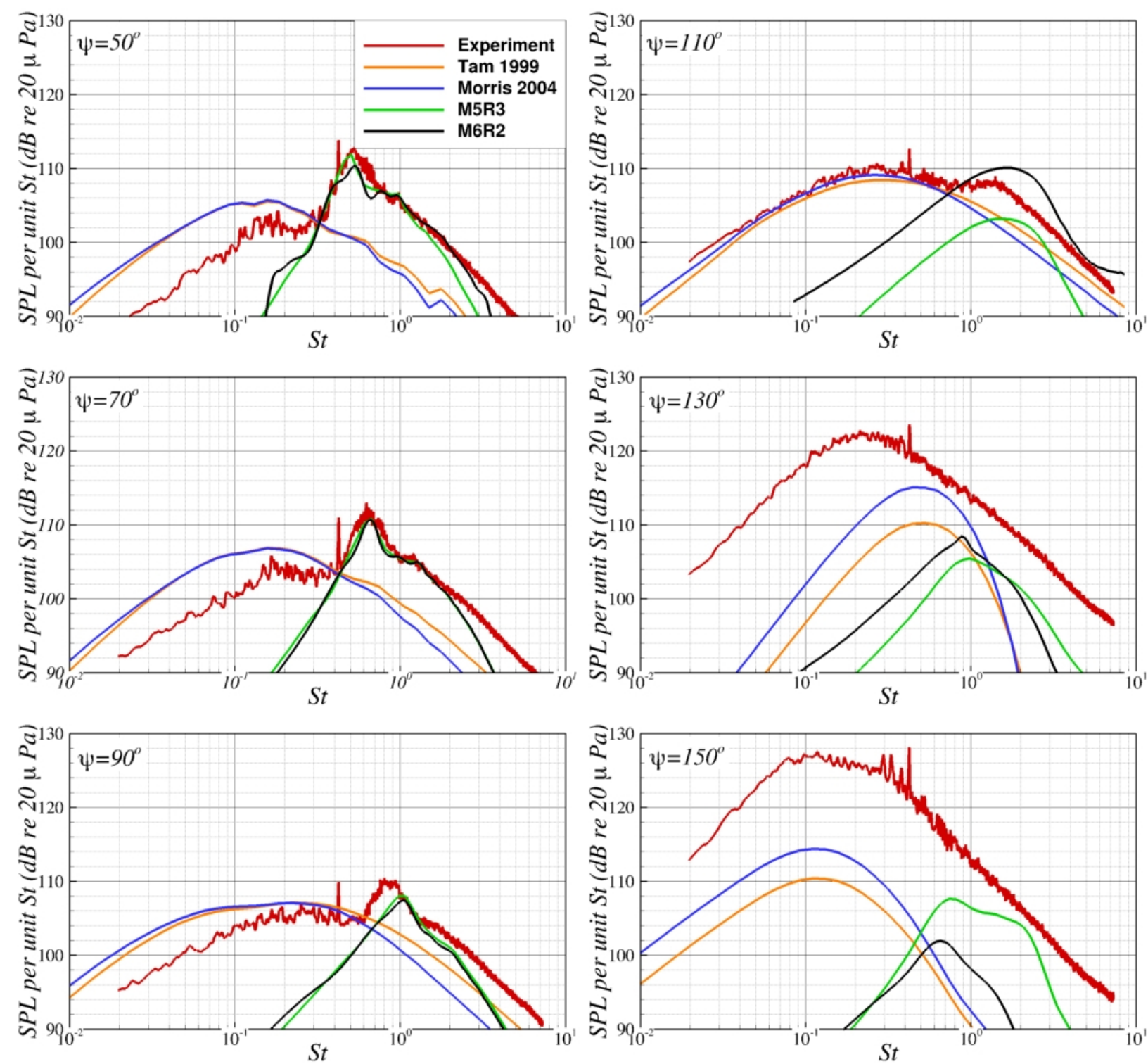

Figure 11. The noise spectra from the SMC000 nozzle case SMC0005, $M_{d}=1.00 D=0.0508 \mathrm{~m}$, with operating conditions $M_{j}=1.24$ and $T T R=2.70$, at a distance of $R / D=100$ and observer angles $\Psi$. 

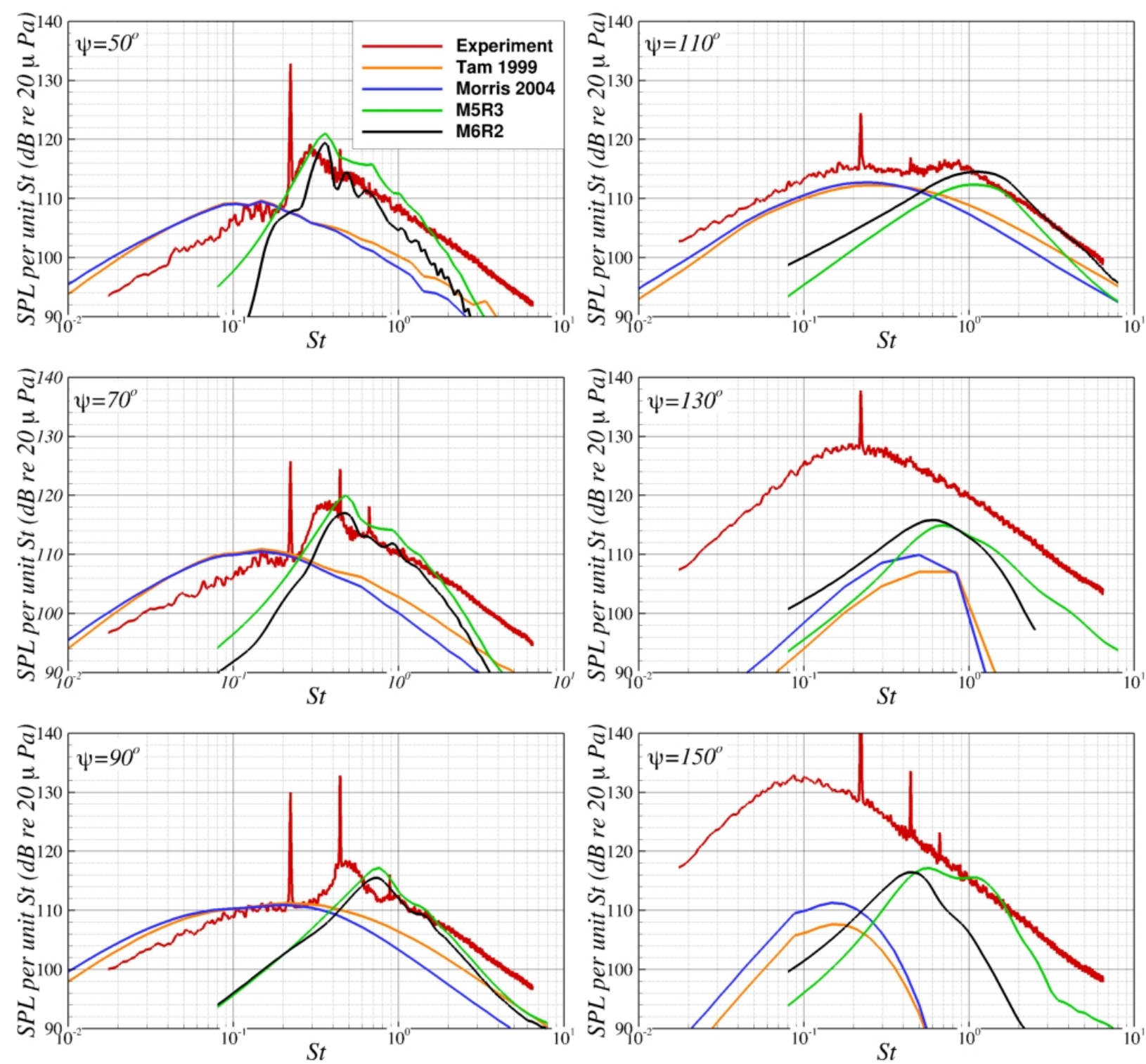

Figure 12. The noise spectra from the SMC000 nozzle case SMC0006, $M_{d}=1.00 D=0.0508$ m, with operating conditions $M_{j}=1.39$ and $T T R=2.70$, at a distance of $R / D=100$ and observer angles $\Psi$. 

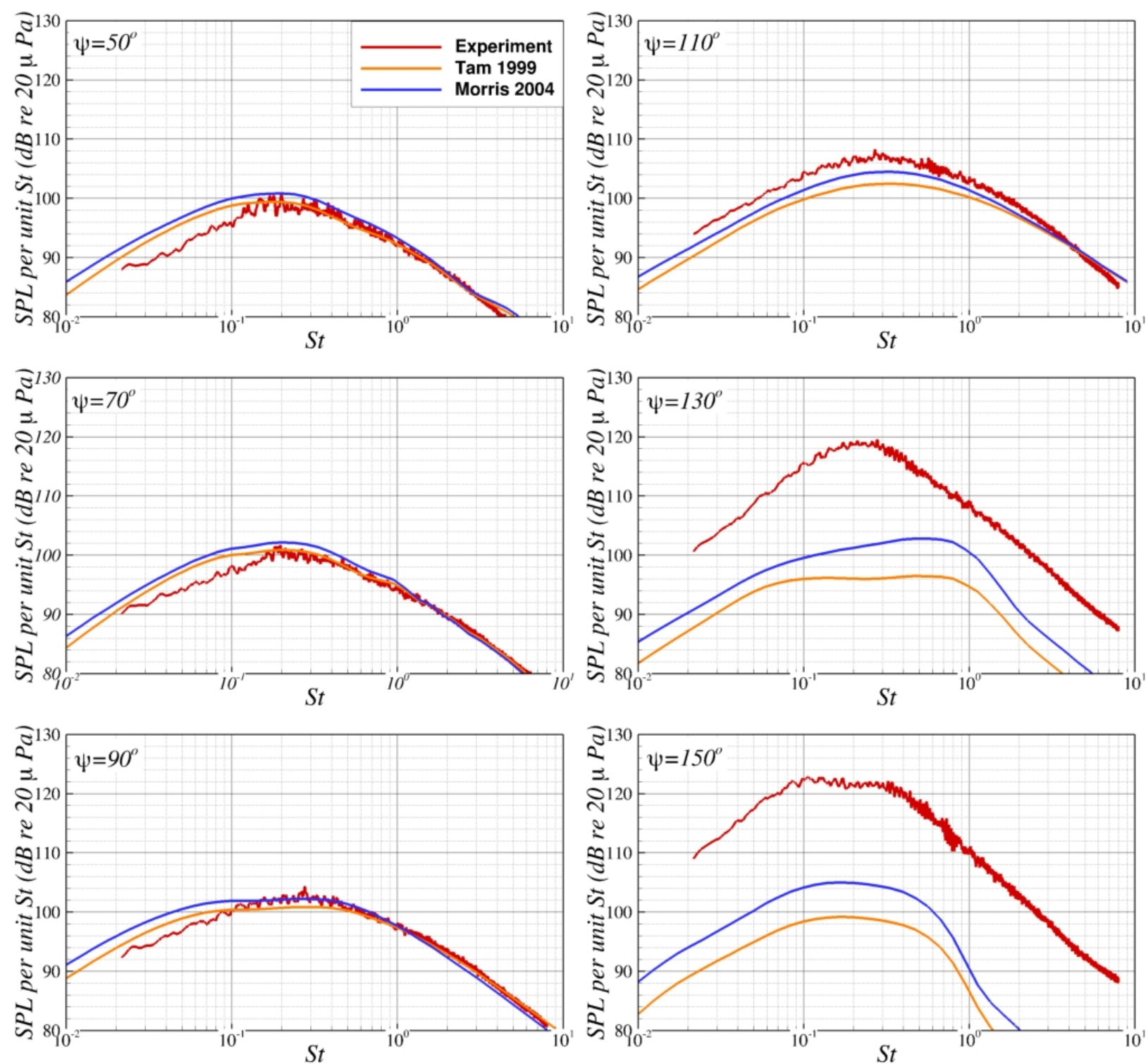

Figure 13. The noise spectra from the SMC000 nozzle case SMC0007, $M_{d}=1.00 D=0.0508 \mathrm{~m}$, with operating conditions $M_{j}=1.00$ and $T T R=3.20$, at a distance of $R / D=100$ and observer angles $\Psi$. 

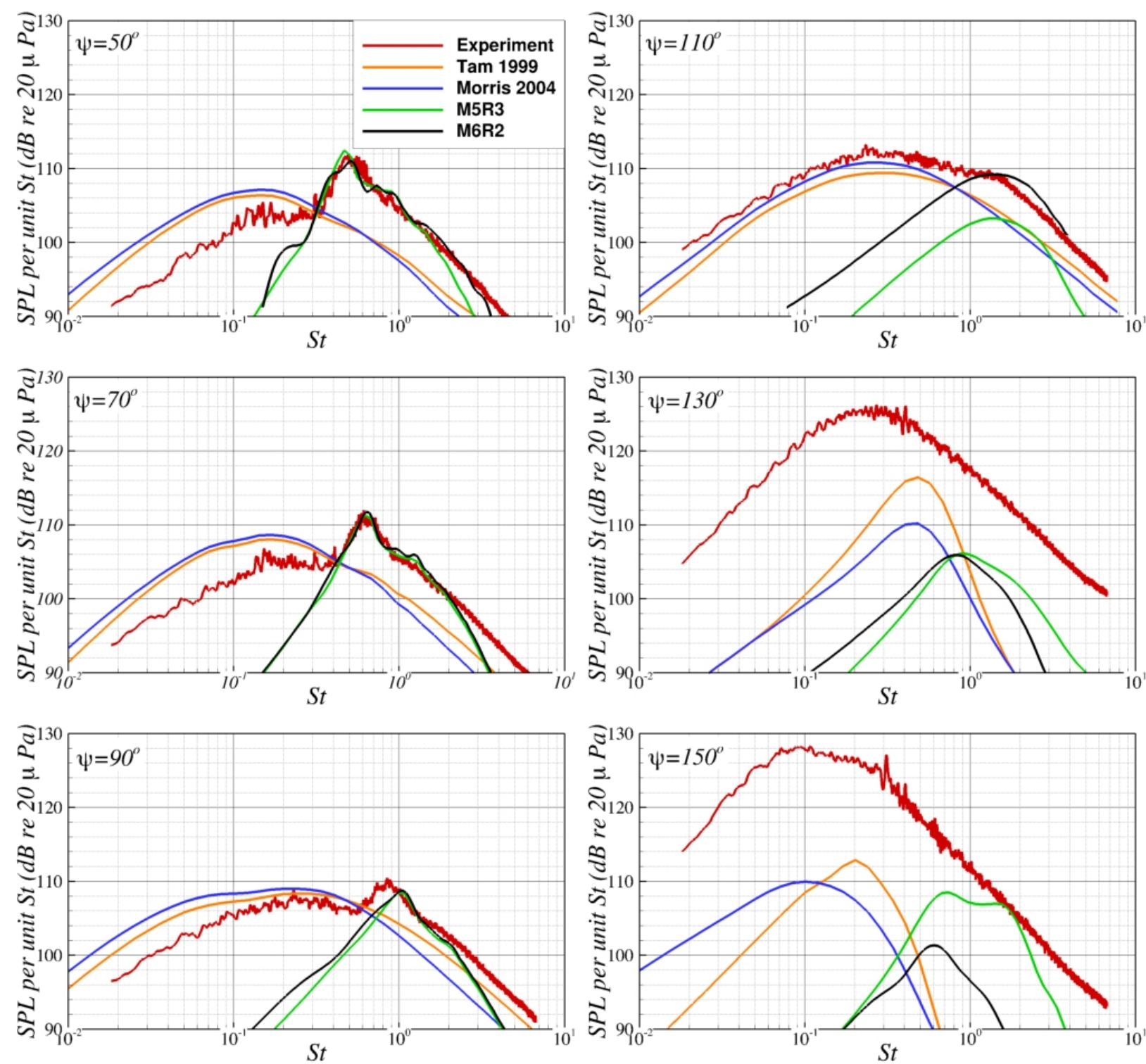

Figure 14. The noise spectra from the SMC000 nozzle case SMC0008, $M_{d}=1.00 D=0.0508 \mathrm{~m}$, with operating conditions $M_{j}=1.24$ and $T T R=2.70$, at a distance of $R / D=100$ and observer angles $\Psi$. 

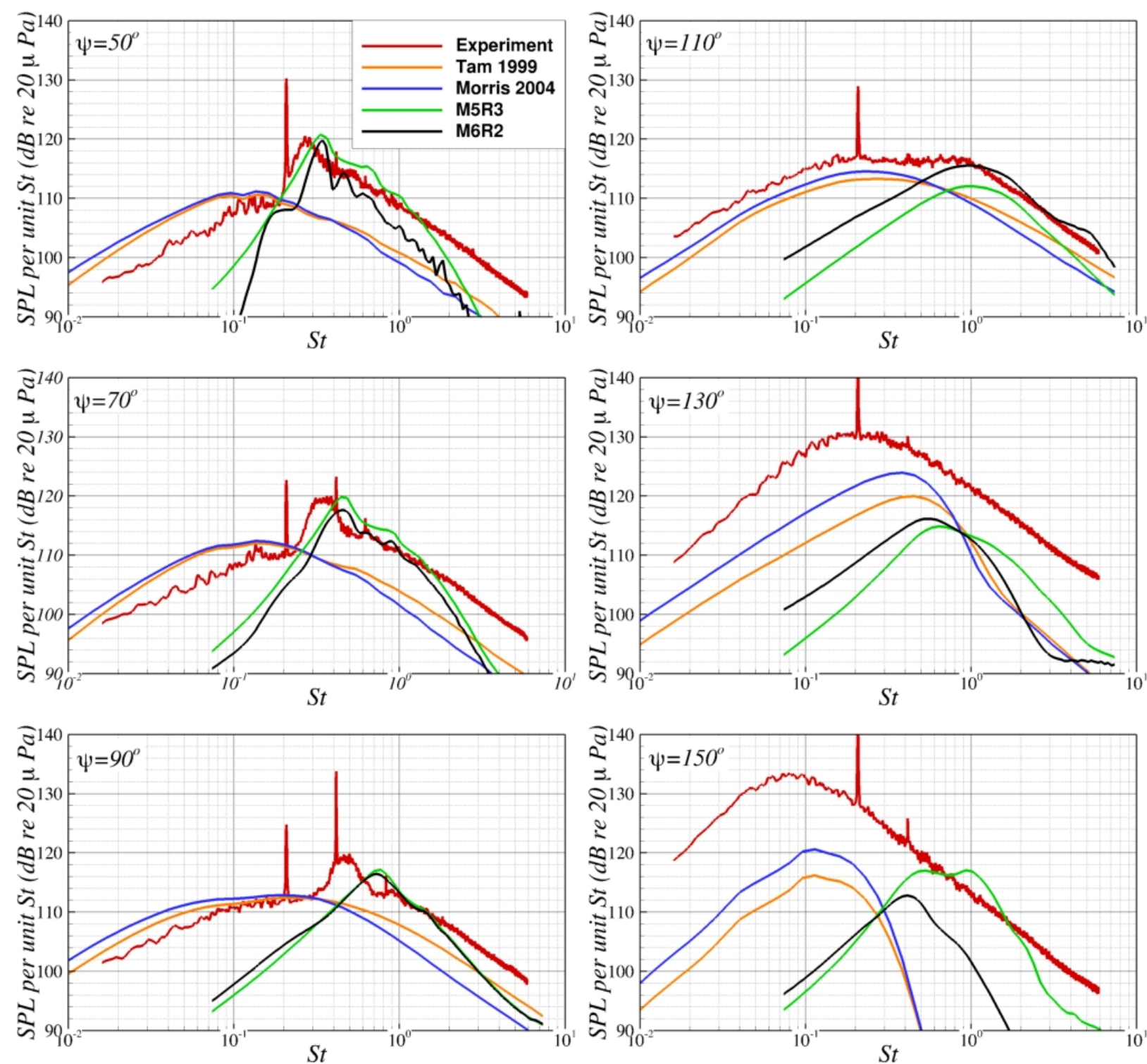

Figure 15. The noise spectra from the SMC000 nozzle case SMC0009, $M_{d}=1.00 D=0.0508 \mathrm{~m}$, with operating conditions $M_{j}=1.39$ and $T T R=3.20$, at a distance of $R / D=100$ and observer angles $\Psi$. 

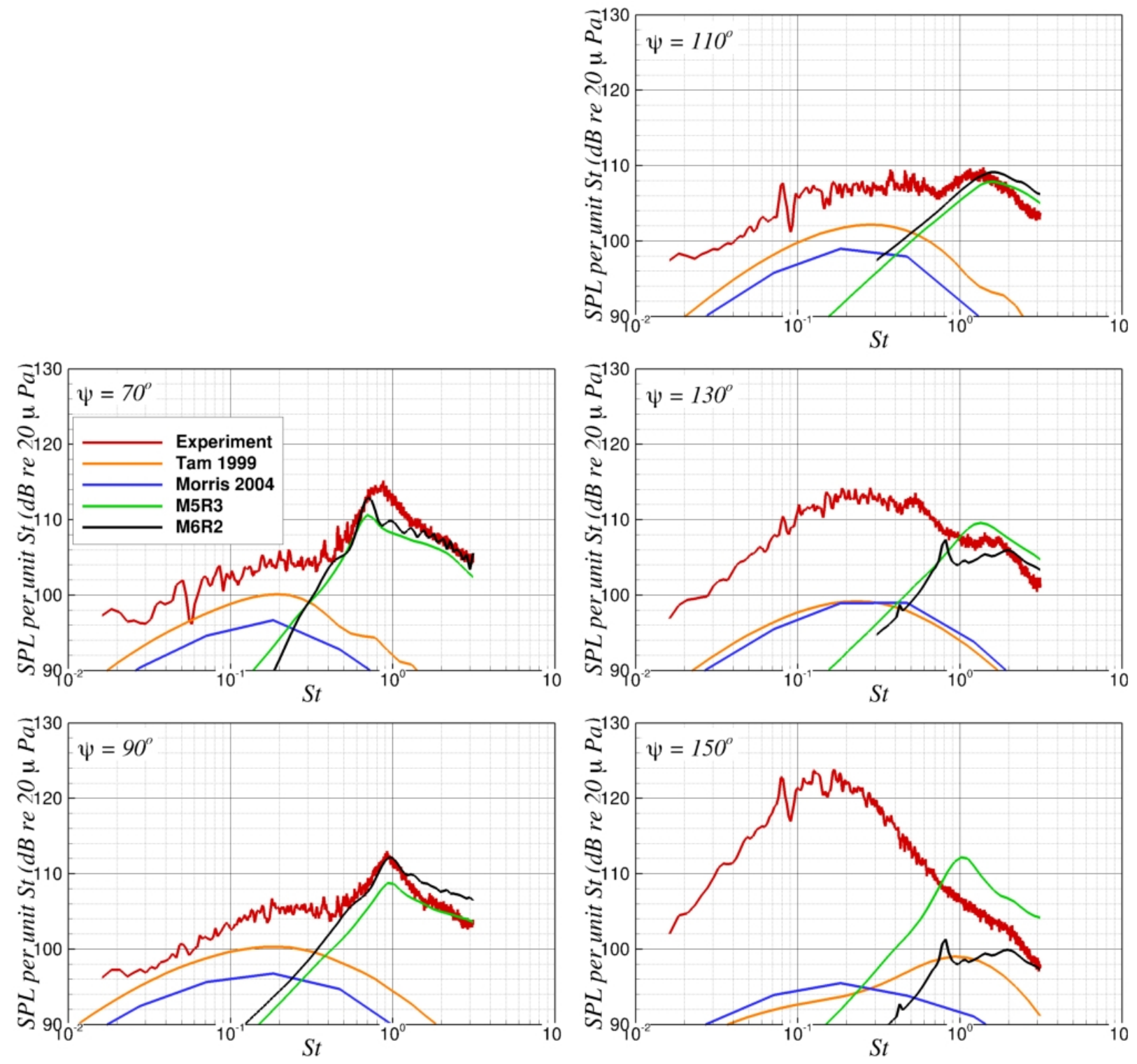

Figure 16. The noise spectra from the PSU000 nozzle case PSU0001, $M_{d}=1.50 D=0.0127$ m, with operating conditions $M_{j}=1.30$ and $T T R=1.00$, at a distance of $R / D=100$ and observer angles $\Psi$. The experimental data is courtesy of Professor D. K. McLaughlin of the Pennsylvania State University. 

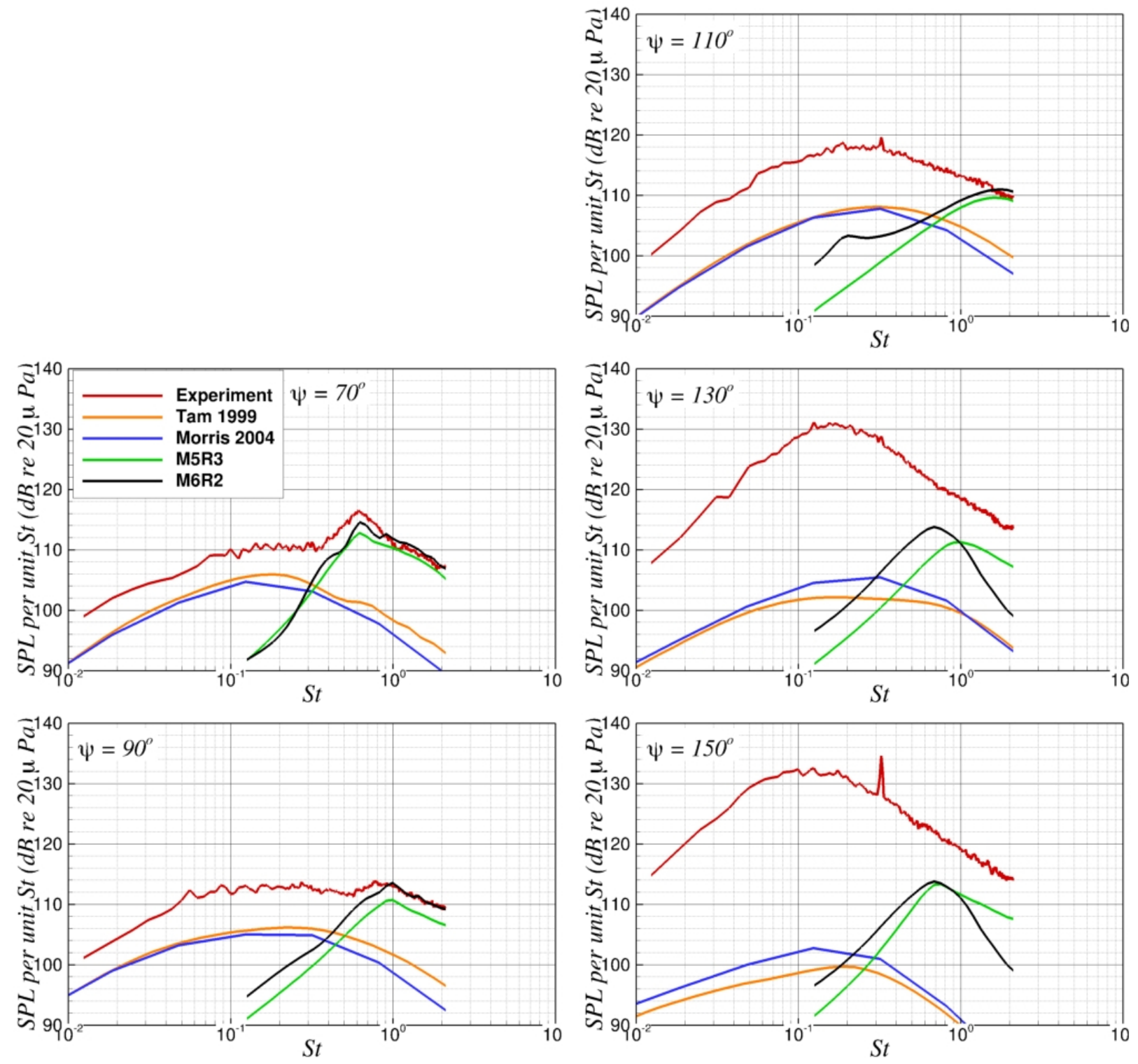

Figure 17. The noise spectra from the PSU000 nozzle case PSU0003, $M_{d}=1.50 D=0.0127$ m, with operating conditions $M_{j}=1.30$ and $T T R=2.20$, at a distance of $R / D=100$ and observer angles $\Psi$. The experimental data is courtesy of Professor D. K. McLaughlin of the Pennsylvania State University. 

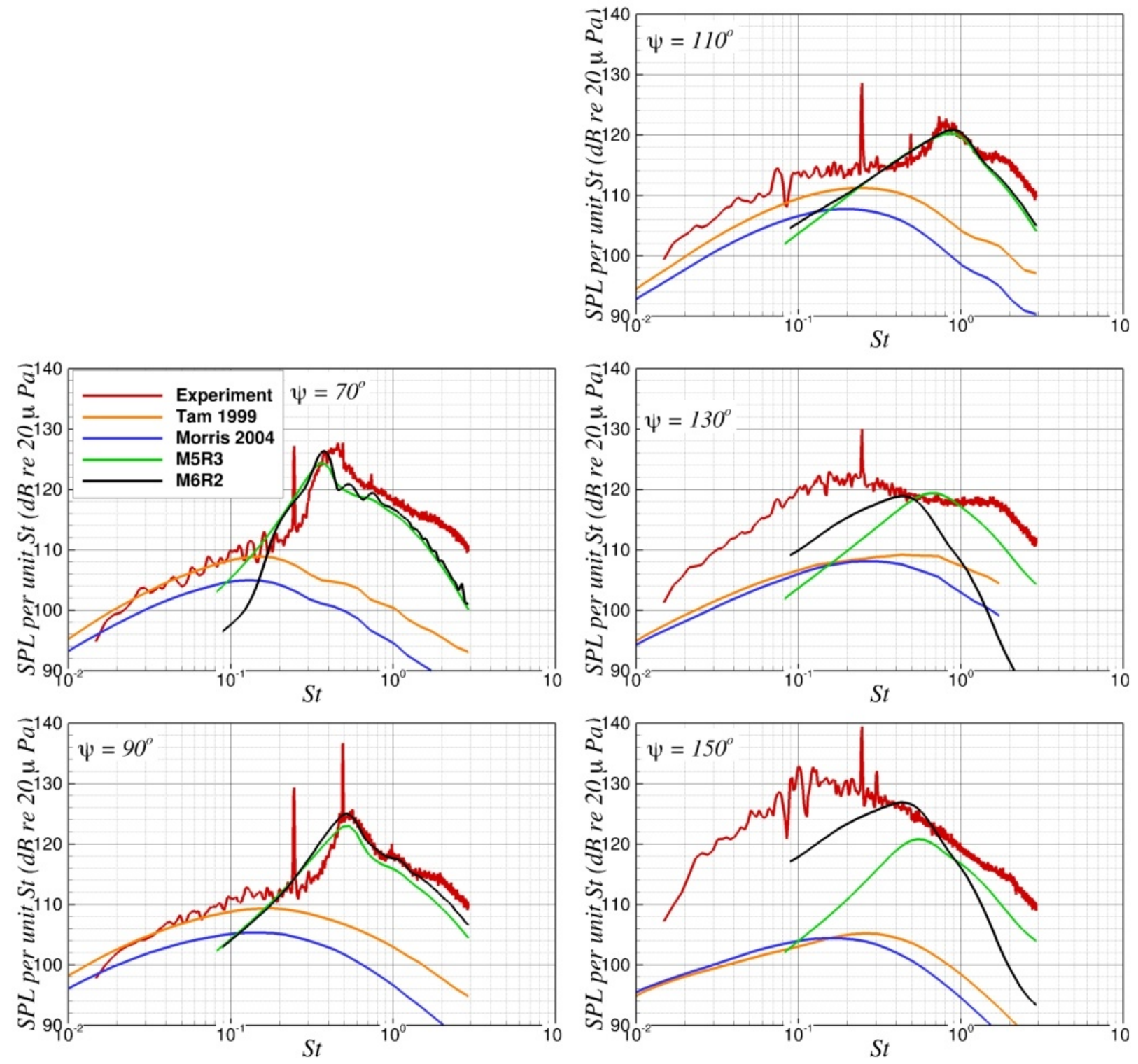

Figure 18. The noise spectra from the PSU000 nozzle case PSU0002, $M_{d}=1.50 D=0.0127$ m, with operating conditions $M_{j}=1.70$ and $T T R=1.00$, at a distance of $R / D=100$ and observer angles $\Psi$. The experimental data is courtesy of Professor D. K. McLaughlin of the Pennsylvania State University. 

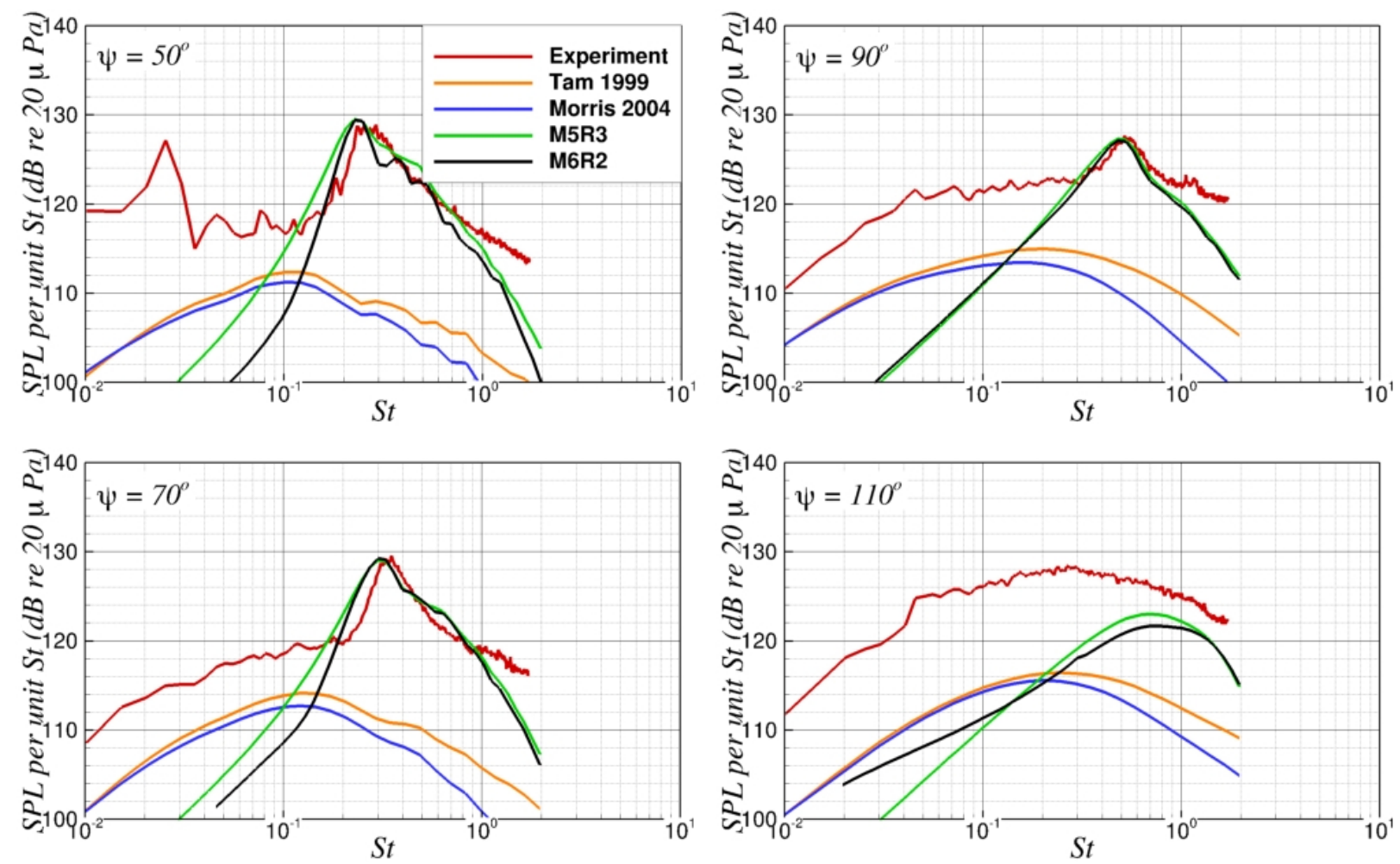

Figure 19. The noise spectra from the PSU000 nozzle case PSU0004, $M_{d}=1.50 D=0.0127 \mathrm{~m}$, with operating conditions $M_{j}=1.70$ and $T T R=2.20$, at a distance of $R / D=100$ and observer angles $\Psi$. The experimental data is courtesy of Professor D. K. McLaughlin of the Pennsylvania State University. 

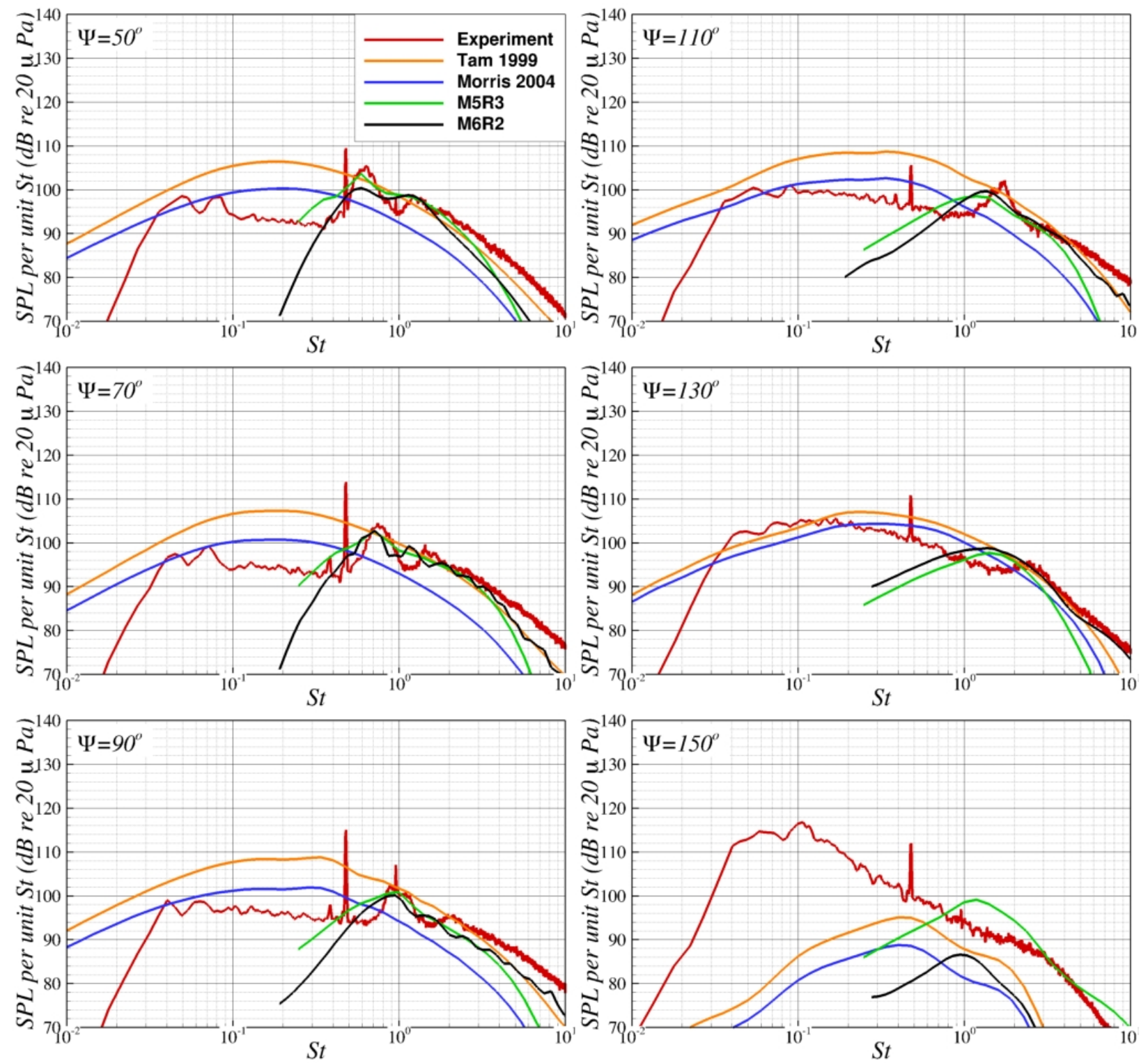

Figure 20. The noise spectra from the Boeing nozzle case Dual4, $M_{d p}=1.00 D_{p}=0.06223 \mathbf{m ~} M_{d s}=1.00 \mathrm{~m}$ $D_{s i}=0.1017 \mathbf{m ~} D_{s o}=0.1525 \mathrm{~m}$, with operating conditions $M_{j p}=0.710 M_{j s}=1.358$ and $T T R_{p}=2.14 T T R_{s}=1.00$, at a distance of $R / D=100$ and observer angles $\Psi$. The experimental data is courtesy of Dr. K. Viswanathan of the Boeing Company. 

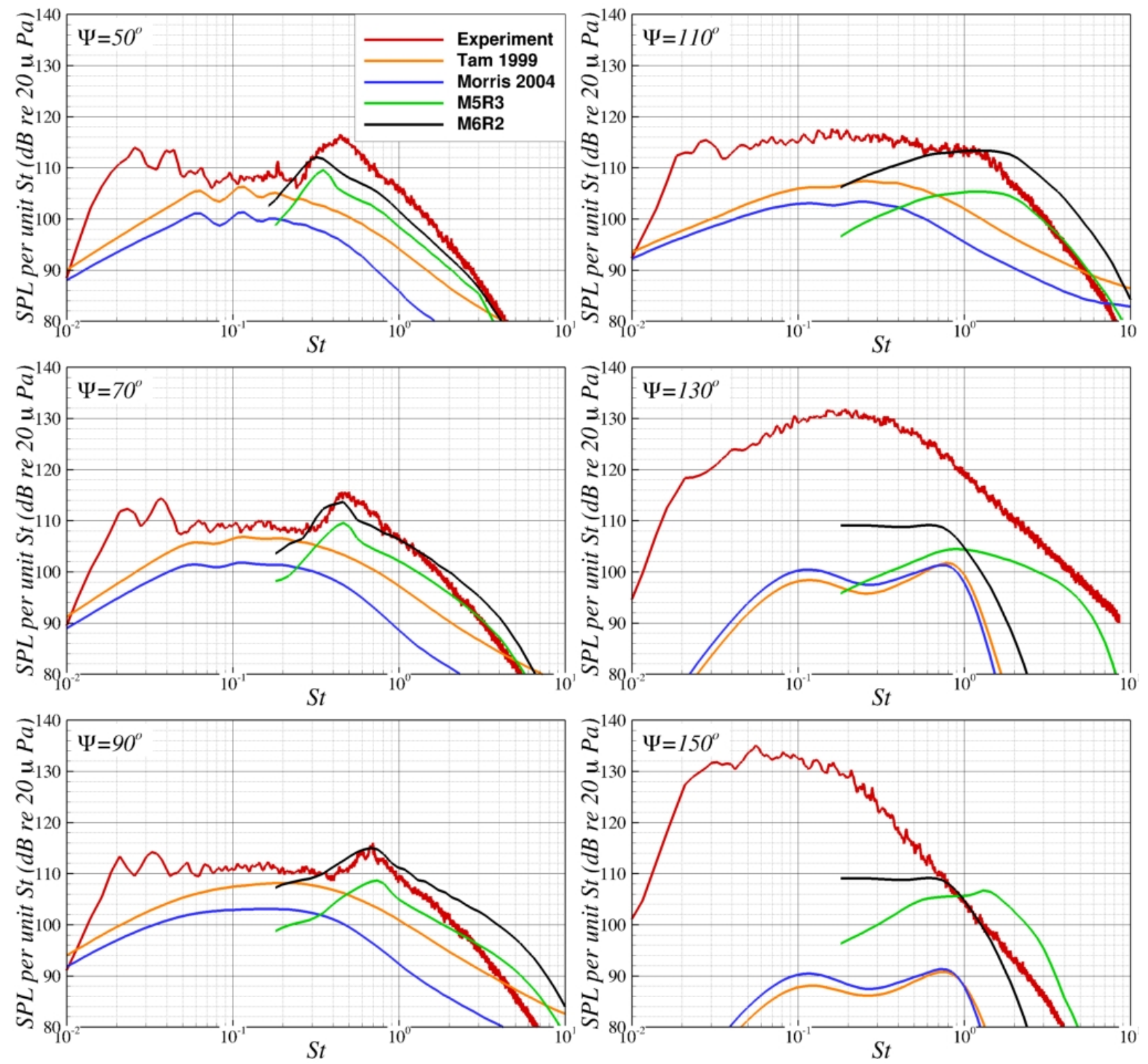

Figure 21. The noise spectra from the Boeing nozzle case Dual5, $M_{d p}=1.00 D_{p}=0.06223 \mathbf{m ~} M_{d s}=1.00 \mathrm{~m}$ $D_{s i}=0.1017 \mathbf{m ~} D_{s o}=0.1525 \mathbf{m}$, with operating conditions $M_{j p}=1.281 M_{j s}=0.848$ and $T T R_{p}=2.870 T T R_{s}=1.00$, at a distance of $R / D=100$ and observer angles $\Psi$. The experimental data is courtesy of Dr. K. Viswanathan of the Boeing Company. 

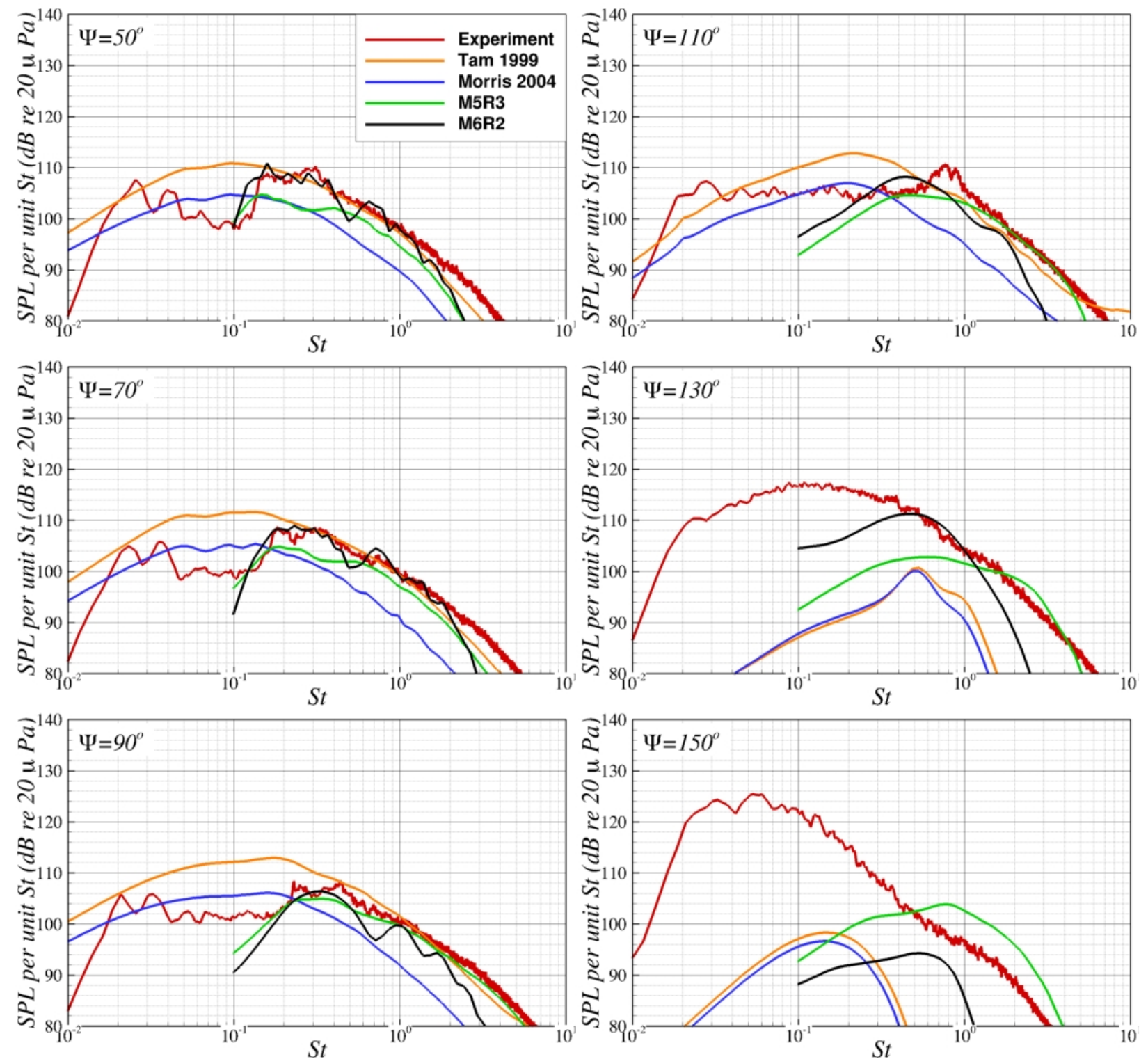

Figure 22. The noise spectra from the Boeing nozzle case Dual7, $M_{d p}=1.00 D_{p}=0.06223 \mathbf{~ m ~} M_{d s}=1.00 \mathrm{~m}$ $D_{s i}=0.1017 \mathbf{m ~} D_{s o}=0.1525 \mathbf{m}$, with operating conditions $M_{j p}=1.281 M_{j s}=1.358$ and $T T R_{p}=2.870 T T R_{s}=1.00$, at a distance of $R / D=100$ and observer angles $\Psi$. The experimental data is courtesy of Dr. K. Viswanathan of the Boeing Company. 